Research Article

\title{
Correlation Measures for Cubic m-Polar Fuzzy Sets with Applications
}

\author{
Harish Garg $\mathbb{D}^{1},{ }^{1}$ Muhammad Riaz $\mathbb{D}^{2},{ }^{2}$ Muhammad Abdullah Khokhar, ${ }^{2}$ and Maryam Saba ${ }^{2}$ \\ ${ }^{1}$ School of Mathematics, Thapar Institute of Engineering and Technology, Deemed University, Patiala 147004, Punjab, India \\ ${ }^{2}$ Department of Mathematics, University of the Punjab, Lahore 54590, Pakistan
}

Correspondence should be addressed to Harish Garg; harishg58iitr@gmail.com

Received 18 April 2021; Accepted 7 July 2021; Published 28 September 2021

Academic Editor: G. Muhiuddin

Copyright (c) 2021 Harish Garg et al. This is an open access article distributed under the Creative Commons Attribution License, which permits unrestricted use, distribution, and reproduction in any medium, provided the original work is properly cited.

A cubic $m$-polar fuzzy set (CmPFS) is a new hybrid extension of $m$-polar fuzzy set and cubic set. A CmPFS is a robust model to express multipolar information in terms of $m$ fuzzy intervals representing membership grades and $m$ fuzzy numbers representing nonmembership grades. In this article, we explore some new operational laws of CmPFSs, produce some related results, and discuss their consequences. We propose relative informational coefficients and relative noninformational coefficients for CmPFSs. These coefficients are analyzed to investigate further properties of CmPFSs. Based on these coefficients, we introduce new correlation measures and their weighted versions for CmPFSs. The value of proposed correlation measures is symmetrical and lies between -1 and 1 . Moreover, the applications of the proposed correlation in pattern recognition and medical diagnosis are developed. The feasibility and efficiency of suggested correlation measures is determined by respective illustrative examples.

\section{Introduction}

Modern systems and logic are the schematic study of the rules that can lead to the acceptance of a certain proposition on the basis of preassumed hypothesis. The development of the modern systems owes to the advancement of set theory and logic. To solve uncertain problems of reallife, the researchers have been established various sets and models. In 1965, Zadeh [1] extended crisp set to fuzzy set (FS) by assigning a real number in interval $[0,1]$ which dictates the membership value of an element/object in the crisp set. Zadeh's pioneer work has a revolutionary importance during the last decades as it laid a foundation to the modern "fuzzy set theory (FST) and its applications." This theory, upon being uplifted to a certain degree, turned to fuzzy logics. Fuzzy logic and fuzzy sets have a large number of applications in computational intelligence, robotics, neural networks, data sciences, and pattern recognition. The idea of fuzzification proved to be an open end for the scientists. A lot of extensions of FST have been introduced so far. The notions of bipolar fuzzy set (BPFS) [2] and intuitionistic fuzzy set (IFS) [3] are both robust extensions of FS. An IFS assigns a pair of membership value and a nonmembership value which lie between 0 and 1 . A BFS is a direct extension of FS which gives information which is bipolar in nature. Its positive membership value $\mu^{+}$ lies in the interval $[0,1]$ and negative membership value $\mu^{-}$ lies in the interval $[-1,0]$. The positive scores depict the consent of a certain property, and the negative scores assigned reflect the consent of a counter property. However, an important point to be noted here is that which type of necessity served as a mother of this extension, that is, which limitation led to this work? The innovative idea of BFS correctly addresses this problem. It should be acknowledged that the individual viewpoint or reasoning is not limited to bipolarity. There can be more than two independent view points to a certain crisp item. For example, in an electoral system, a vote being casted may have exact degree from $[0,1]^{m}$. These degrees may be assigned on the basis of personal mindset, honesty of the candidate, leadership qualities, education, and future plans. Therefore, bipolar fuzzy theory cannot answer this sort of problem and there is a need to invent a new extension. To address this limitation, $m$-polar fuzzy set (mPFS) theory has been 
proposed by Chen et al. [4]. An $m$-polar fuzzy set assigns $m$ independent values/degrees to each crisp element/object.

Jun et al. [5] introduced the concept of cubic set (CS) as an extension of intuitionistic fuzzy set (IFS). A CS assigns a membership grade which is a fuzzy interval and nonmembership which is fuzzy value to each crisp element/ object. Besides, both types of grades are condition free. However, CS allows each of its alternatives to follow one attribute only. Multiattributed information is denied in CS theory. This is the main reason and answer to the important question "why cubic $m$-polar fuzzy set (CmPFS) theory has been developed." The CmPFS (initiated by Riaz and Hashmi [6]) is a new hybrid structure of CS and $m$ PFS, and it manipulates not only multiattributed but also cubic information as well. Therefore, CS and $m$ PFS are the special cases of CmPFS.

Some of the important extensions of fuzzy set are Pythagorean fuzzy set (PFS) [7], q-rung orthopair fuzzy set (q-ROFS) [8], neutrosophic set [9], single-valued neutrosophic set [10], spherical fuzzy set [11-13], and picture fuzzy sets (PicFSs) [14]. Soft set [15] is a robust independent extension of crisp sets for modeling uncertainty problems. Wang et al. [16] introduced Pythagorean fuzzy interactive Hamacher power aggregation operators for assessment of express service quality with entropy weight. Liu et al. [17] suggested Banzhaf-Choquet copula-based aggregation operators for managing q-ROF information. Akram et al. [18] established complex spherical fuzzy prioritized weighted aggregation operators. Sitara et al. [19] proposed novel decision-making analysis based on q-rung picture fuzzy graph structures. Garg $[20,21]$ developed MAGDM process based on operators for intuitionistic multiplicative set and interval-valued picture uncertain linguistic generalized Hamacher aggregation operators. Liu et al. [22] proposed group decision-making using complex q-ROF Bonferroni mean. Liu and Wang [23] established a new MAGDM method based on IF Einstein interactive operations.

Recently, Riaz et al. [24, 25] introduced some new extensions of fuzzy sets named as linear Diophantine fuzzy set (LDFS) and spherical linear Diophantine sets. Kamaci [26] introduced algebraic structure on LDFS with an interesting application to coding theory which is based on LDFS codes. Ayub et al. [27] introduced LDF-relations and their algebraic properties with decision-making application. Almagrabi et al. [28] presented a new approach to the q-LDF emergency decision support system for COVID-19.

Correlation and similarity measures are significant mathematical tools to deal with real-life problems. The development of fuzzy set theories give rise to new extension of correlation and similarity measures. The researchers have been used these measures to develop new techniques for decision-making, pattern recognition, and cluster analysis. Ganie and Singh [29] used similarity measures for MCDM in picture fuzzy environment. Mahmood and Ali [30] used correlation measures to propose the extended TOPSIS method for Pythagorean fuzzy environment. Zulqarnain et al. [31] proposed correlated TOPSIS for the mask selection.
Correlation is a statistic that assesses the extent to which two objects move in relation to each other whereas similarity measure is a function which measures the closeness of two objects. Correlation gives some important applications to statistical analysis. Statistics has effectively used probability methods for dealing with many real space issues focused on random processing of data. Similarly, some statistical problems in engineering sciences have also been addressed by the same probabilistic approaches. However, these approaches have some drawbacks, since these methods are based on random sample selection to obtain a certain degree of trust. When the data are collected on a large-scale, massive fuzziness is involved therein which makes it difficult to optimize the idealistic probability. The main question is that how to overcome such obstacles to obtain idealistic results? The answer to this question is somewhat complicated. The first thing to be noted is that in every real-world problem, no matter it is an engineering, medical, or administrative issue, uncertainty always finds its place. In statistics, correlation is one of the most important concepts we encounter with and is digested by many other subjects such as economics, finance, engineering, and management sciences. However, the problem is that this type of correlation can cope with the crisp data only and we have been managing many real-life problems with the help of this without taking into account uncertainty. Since the birth of fuzzy set, many uncertain settings have been introduced to tackle variability. We have seen above that a lot of ambiguous sets have been defined so far to handle imprecise information. Therefore, there is a need to introduce correlation measures in every environment because a real problem may be diverse in its uncertain nature. To address this issue, Ejegwa et al. [32], Gerstenkorn and Mańko [33], and Szmidt and Kacprzyk [34] proposed some correlation measures for IFSs. Garg et al. [35] presented the correlation coefficient for cubic intuitionistic fuzzy set and hence presented a decision-making algorithm. Correlation measures are used in PFSs. Lin et al. [36] and Thao [37] also discussed limitations of the previously defined correlations and put forward new directional correlations for PFSs.

The main objectives of the manuscript are listed as follows:

(1) A cubic $m$-polar fuzzy set (CmPFS) is a robust fusion of cubic sets and $m$-polar fuzzy set to solve more complicated decision-making problems in the multipolar and cubic environment. This model is more suitable to express vague information efficiently, and the decision-makers find more flexibility to give their assessment towards multicriterion, multiattribute, multisensor, multisystems, and multirelations.

(2) To define new operations on CmPFSs and discuss related results.

(3) To develop relative informational coefficients and relative noninformational coefficients for CmPFSs.

(4) To analyze these coefficients to investigate further properties of CmPFSs. 
(5) To define new correlation measures and their weighted versions for CmPFSs.

(6) To construct new algorithms with the help of new correlation measures for solving the complicated problems of pattern recognition and medical diagnosis which cannot be tackled by existing techniques. The feasibility and efficiency of suggested correlation measures is determined by respective illustrative examples.

Figure 1 demonstrates novel hybrid extension of cubic set and $m$-polar fuzzy set named as cubic $m$-polar fuzzy set.

This paper is organized as follows. In Section 2, we first recall few basic concepts of fuzzy sets, cubic sets, and $m$-polar fuzzy sets. Then, we review definition and operations of CmPFSs. In Section 3, we discuss some novel features of CmPFSs depending on new operations. We also define support, core, height, level sets, and binary relations. In Section 4, we define correlation measures for CmPFSs. Their weighted versions have also been addressed. We prove that all types of correlation measures are symmetrical and lie in the interval $[-1,1]$, the correlation of two CmPFSs is 1 only if either both sets are equal or one of the sets is a positive linear multiple of the other, and the correlation of the two sets is -1 only if one set is a positive linear multiple of the complement of the other set. In Section 5, new algorithms are proposed using proposed correlation measures under $\mathrm{CmPF}$ environment for solving the complicated problems of pattern recognition and medical diagnosis. The feasibility and efficiency of suggested correlation measures is determined by respective illustrative examples. The conclusion of this research studies is presented in Section 6.

\section{Preliminaries}

Some basic definitions and operations are discussed here in this section which can serve foundation for current research work. We shall use $\aleph$ as a universal space.

Definition 1 (see [1]). A fuzzy set (FS) on a universe $\aleph$ is a rule which assigns a number from the interval $[0,1]$ to each element in $\aleph$. This number is referred to as membership grade of the crisp element. Mathematically, an FS is described by the membership function $\mu: \aleph \longrightarrow[0,1]$.

Definition 2 (see [4]). An $m$-polar fuzzy set ( $m$ PFS) on a universe $\mathcal{N}$ is defined by

$$
\begin{aligned}
\mathfrak{M} & =\left\{\left\langle\kappa,\left(\mu_{i}(\kappa)\right)_{i=1}^{m}\right\rangle: \kappa \in \aleph\right\} \\
& =\left\{\left\langle\kappa, \mu_{1}(\kappa), \mu_{2}(\kappa), \ldots, \mu_{m}(\kappa)\right\rangle: \kappa \in \aleph\right\},
\end{aligned}
$$

where $\mu_{i}:[0,1]^{m} \longrightarrow[0,1] ; i=1,2, \ldots, m$, is the $i^{\text {th }}$ projection mapping.

An $m$-polar fuzzy set is a direct extension of FS that describes the multipolarity of the objects. An $m$ PFS assigns $m$ fuzzy grades to each object/element of universe $\aleph$.

Definition 3 (see [5]). A cubic set (CS) is defined by a rule which assigns to each element/object of the discourse $\aleph$ a

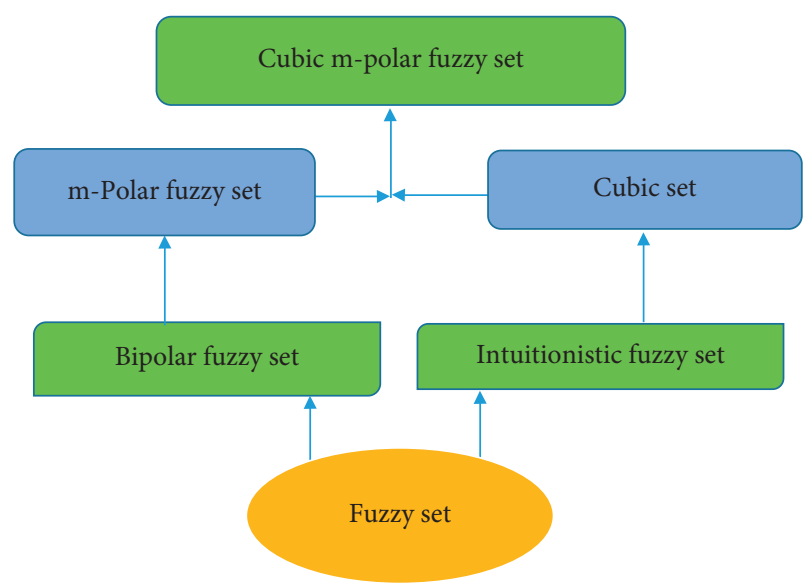

Figure 1: Cubic $m$-polar fuzzy set: a hybrid model.

subinterval of $[0,1]$ called membership value which is a fuzzy subinterval and a nonmembership value from $[0,1]$. Mathematically, we can write

$$
\operatorname{CS}(\aleph)=\left\{\left(\kappa, A_{\kappa}, B_{\kappa}\right): \kappa \in \aleph\right\},
$$

where $A_{\kappa}$ is a fuzzy subinterval and $B_{\kappa}$ is a fuzzy value assigned to the alternative $\kappa$. There is no restriction on $B_{\kappa}$ to be a number from $A_{\kappa}$. If, however, $B_{\kappa} \in A_{\kappa}$, then $\operatorname{CS}(\aleph)$ is called internal cubic set (ICS). Otherwise, it is called external cubic set (ECS). Thus, cubic information is of two categories, that is, internal and external. So, whenever we deal with cubic sets, we must ensure its behaviour.

Definition 4 (see [6]). A cubic m-polar fuzzy set (CmPFS) is a set which deals with multipolar information in cubic environment. It assigns to each member of the crisp discourse a cubic element allowing multipolarity. If we take $\aleph$ as universe of discourse, the CmPFS can be viewed mathematically as follows:

$$
\operatorname{CmPFS}(\aleph)=\left\{\left\langle\kappa,\left[\eta_{i}^{-}(\kappa), \eta_{i}^{+}(\kappa)\right], \eta_{i}(\kappa)\right\rangle_{i=1}^{m}\right\} .
$$

For convenience, the term $\left\langle\left[\eta_{j}^{-}, \eta_{j}^{+}\right], \eta_{j}\right\rangle_{j=1}^{m}$ is referred to as cubic $m$-polar fuzzy number (CmPFN).

2.1. Operations on CmPFSs. Let $A_{\aleph}^{(1)}=\left\{\left\langle\kappa,\left[\eta_{i}^{-}(\kappa), \eta_{i}^{+}(\kappa)\right]\right.\right.$, $\left.\left.\eta_{i}(\kappa)\right\rangle_{i=1}^{m}: \kappa \in \aleph\right\}$ and $A_{\aleph}^{(2)}=\left\{\left\langle\kappa,\left[\zeta_{i}^{-}(\kappa), \zeta_{i}^{+}(\kappa)\right], \zeta_{i}(\kappa)\right\rangle_{i=1}^{m}\right.$ : $\kappa \in \aleph\}$ be the CmPFSs. Then, the operations for these sets under P-ordering are defined as follows:

(1) $A_{\aleph}^{(1)}=A_{\aleph}^{(2)} \Leftrightarrow \eta_{i}^{-}(\kappa)=\zeta_{i}^{-}(\kappa), \eta_{i}^{+}(\kappa)=\zeta_{i}^{+}(\kappa)$ and $\eta_{i}(\kappa)=\zeta_{i}(\kappa)$. For all $i=1,2, \ldots, m$ and $\kappa \in \aleph$

(2) $\left(A_{\aleph}^{(1)}\right)^{c}=\left\{\left\langle\kappa,\left[1-\eta_{i}^{+}(\kappa), 1-\eta_{i}^{-}(\kappa)\right], 1-\eta_{i}(\kappa)\right\rangle_{i=1}^{m}: \kappa\right.$ $\in \aleph\}$

(3) $A_{\aleph}^{(1)} \cup_{P} A_{\aleph}^{(2)}=\left\{\left\langle\kappa,\left[\eta_{i}^{-}(\kappa) \vee \zeta_{i}^{-}(\kappa), \eta_{i}^{+}(\kappa) \vee \zeta_{i}^{+}(\kappa)\right], \eta_{i}\right.\right.$ $\left.\left.(\kappa) \vee \zeta_{i}(\kappa)\right\rangle_{i=1}^{m}: \kappa \in \aleph\right\}$

(4) $A_{\aleph}^{(1)} \cap_{P} A_{\aleph}^{(2)}=\left\{\left\langle\kappa,\left[\eta_{i}^{-}(\kappa) \wedge \zeta_{i}^{-}(\kappa), \eta_{i}^{+}(\kappa) \wedge \zeta_{i}^{+}(\kappa)\right], \eta_{i}\right.\right.$ $\left.\left.(\kappa) \wedge \zeta_{i}(\kappa)\right\rangle_{i=1}^{m}: \kappa \in \aleph\right\}$

(5) $A_{\aleph}^{(1)} \subseteq_{P} A_{\aleph}^{(2)} \Leftrightarrow \eta_{i}^{-}(\kappa) \leq \zeta_{i}^{-}(\kappa), \eta_{i}^{+}(\kappa) \leq \zeta_{i}^{+}(\kappa)$ and $\eta_{i}(\kappa)$ $\leq \zeta_{i}(\kappa)$. For all $i=1,2, \ldots, m$ and $\kappa \in \aleph$ 
(6) $A_{\aleph}^{(1)} \oplus_{P} A_{\aleph}^{(2)}=\left\{\left\langle\kappa,\left[\eta_{i}^{-}(\kappa)+\zeta_{i}^{-}(\kappa)-\eta_{i}^{-}(\kappa) \zeta_{i}^{-}(\kappa), \eta_{i}^{+}\right.\right.\right.$ $\left.(\kappa)+\zeta_{i}^{+}(\kappa)-\eta_{i}^{+}(\kappa) \zeta_{i}^{+}(\kappa)\right], \eta_{i}(\kappa)+\zeta_{i}(\kappa)-\eta_{i}(\kappa) \zeta_{i}$ $\left.(\kappa)\rangle_{i=1}^{m}: \kappa \in \aleph\right\}$

(7) $A_{\aleph}^{(1)} \otimes_{P} A_{\aleph}^{(2)}=$

$\left\{\left\langle\kappa,\left[\eta_{i}^{-}(\kappa) \zeta_{i}^{-}(\kappa), \eta_{i}^{+}(\kappa) \zeta_{i}^{+}(\kappa)\right], \eta_{i}(\kappa) \zeta_{i}(\kappa)\right\rangle_{i=1}^{m}: \kappa \in \aleph\right\}$

(8) $\varsigma A_{\aleph}^{(1)}=\left\{\left\langle\kappa,\left[1-\left(1-\eta_{i}^{-}(\kappa)\right)^{\varsigma}, 1-\left(1-\eta_{i}^{+}(\kappa)\right)^{\varsigma}\right], 1\right.\right.$ $\left.\left.-\left(1-\eta_{i}(\kappa)\right)^{\varsigma}\right\rangle_{i}=1^{m}: \kappa \in \aleph\right\}$

(9) $\left(A_{\aleph}^{(1)}\right)^{\varsigma}=\left\{\left\langle\kappa,\left[\left(\eta_{i}^{-}(\kappa)\right)^{\varsigma},\left(\eta_{i}^{+}(\kappa)\right)^{\varsigma}\right],\left(\eta_{i}(\kappa)\right)^{\varsigma}\right\rangle_{i=1}^{m}\right.$ : $\kappa \in \aleph\}$

The R-ordering operations for the abovementioned CmPFSs are given as follows:

(Note: the equality and complement operations for these sets are universal; that is, they are independent or $\mathrm{P} / \mathrm{R}$ ordering.)

(1) $A_{\aleph}^{(1)} \cup_{R} A_{\aleph}^{(2)}=\left\{\left\langle\kappa,\left[\eta_{i}^{-}(\kappa) \vee \zeta_{i}^{-}(\kappa), \eta_{i}^{+}(\kappa) \vee \zeta_{i}^{+}\right.\right.\right.$ $\left.\left.(\kappa)], \eta_{i}(\kappa) \wedge \zeta_{i}(\kappa)\right\rangle_{i}=1^{m}: \kappa \in \aleph\right\}$

(2) $A_{\aleph}^{(1)} \cap_{R} A_{\aleph}^{(2)}=\left\{\left\langle\kappa,\left[\eta_{i}^{-}(\kappa) \wedge \zeta_{i}^{-}(\kappa), \eta_{i}^{+}(\kappa) \wedge \zeta_{i}^{+}(\kappa)\right]\right.\right.$, $\left.\left.\eta_{i}(\kappa) \vee \zeta_{i}(\kappa)\right\rangle_{i=1}^{m}: \kappa \in \aleph\right\}$

(3) $A_{\aleph}^{(1)} \subseteq_{R} A_{\aleph}^{(2)} \Leftrightarrow \eta_{i}^{-}(\kappa) \leq \zeta_{i}^{-}(\kappa), \eta_{i}{ }^{+}(\kappa) \leq \zeta_{i}^{+}(\kappa)$ and $\eta_{i}$ $(\kappa) \geq \zeta_{i}(\kappa)$. For all $i=1,2, \ldots, m$ and $\kappa \in \aleph$.

(4) $A_{\aleph}^{(1)} \oplus_{R} A_{\aleph}^{(2)}=\left\{\left\langle\kappa,\left[\eta_{i}^{-}(\kappa)+\zeta_{i}^{-}(\kappa)-\eta_{i}^{-} \quad(\kappa) \zeta_{i}^{-}(\kappa)\right.\right.\right.$, $\left.\left.\left.\eta_{i}^{+}(\kappa)+\zeta_{i}^{+}(\kappa)-\eta_{i}^{+}(\kappa) \zeta_{i}^{+}(\kappa)\right], \eta_{i}(\kappa) \zeta_{i}(\kappa)\right\rangle_{i=1}^{m}: \kappa \in \aleph\right\}$

(5) $A_{\aleph}^{(1)} \otimes_{R} A_{\aleph}^{(2)}=\left\{\left\langle\kappa,\left[\eta_{i}^{-}(\kappa) \zeta_{i}^{-}(\kappa), \eta_{i}^{+}(\kappa) \zeta_{i}^{+}(\kappa)\right], \eta_{i}(\kappa)+\right.\right.$ $\left.\left.\zeta_{i}(\kappa)-\eta_{i}(\kappa) \zeta_{i}(\kappa)\right\rangle_{i=1}^{m}: \kappa \in \aleph\right\}$

(6) $\varsigma A_{\aleph}^{(1)}=\left\{\left\langle\kappa,\left[1-\left(1-\eta_{i}^{-}(\kappa)\right)^{\varsigma}, 1-\left(1-\eta_{i}^{+}(\kappa)\right)^{\varsigma}\right]\right.\right.$, $\left.\left.\left(\eta_{i}(\kappa)\right)^{\varsigma}\right\rangle_{i=1}^{m}: \kappa \in \aleph\right\}$

(7) $\left(A_{\aleph}^{(1)}\right)^{\varsigma}=\left\{\left\langle\kappa,\left[\left(\eta_{i}^{-}(\kappa)\right)^{\varsigma},\left(\eta_{i}^{+}(\kappa)\right)^{\varsigma}\right], 1-\left(1-\eta_{i}\right.\right.\right.$ $\left.\left.(\kappa))^{\varsigma}\right\rangle_{i=1}^{m}: \kappa \in \aleph\right\}$

Note: it is interesting to note that the collection of all CmPFSs on a universe $\aleph$ is closed under these operations.

\section{Some Novel Features of CmPFSs}

In this section, we give some properties and related results for CmPFSs.

Theorem 1. Let $A_{\aleph}^{(1)}, A_{\aleph}^{(2)}$, and $A_{\aleph}^{(3)}$ be CmPFSs. Then,

(1) $A_{\aleph}^{(1)} \cup_{P} A_{\aleph}^{(2)}=A_{\aleph}^{(2)} \cup_{P} A_{\aleph}^{(1)}$

(2) $A_{\aleph}^{(1)} \cap_{P} A_{\aleph}^{(2)}=A_{\aleph}^{(2)} \cap_{P} A_{\aleph}^{(1)}$

(3) $A_{\aleph}^{(1)} \subseteq_{P} A_{\aleph}^{(2)}$ and $A_{\aleph}^{(2)} \subseteq_{P} A_{\aleph}^{(3)}$ implies $A_{\aleph}^{(1)} \subseteq_{P} A_{\aleph}^{(3)}$

(4) $A_{\aleph}^{(1)} \cup_{P}\left(A_{\aleph}^{(2)} \cup_{P} A_{\aleph}^{(3)}\right)=\left(A_{\aleph}^{(1)} \cup_{P} A_{\aleph}^{(2)}\right) \cup_{P} A_{\aleph}^{(3)}$

(5) $A_{\aleph}^{(1)} \cap_{P}\left(A_{\aleph}^{(2)} \cap_{P} A_{\aleph}^{(3)}\right)=\left(A_{\aleph}^{(1)} \cap_{P} A_{\aleph}^{(2)}\right) \cap_{P} A_{\aleph}^{(3)}$

(6) $A_{\aleph}^{(1)} \cup_{P}\left(A_{\aleph}^{(2)} \cap_{P} A_{\aleph}^{(3)}\right)=\left(A_{\aleph}^{(1)} \cup_{P} A_{\aleph}^{(2)}\right) \cap_{P}\left(A_{\aleph}^{(1)} \cup_{P}\right.$

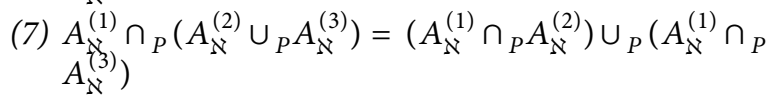

Proof. Let $A_{\aleph}^{(1)}=\left\{\left\langle\kappa,\left[\eta_{i}^{-}(\kappa), \eta_{i}^{+}(\kappa)\right], \eta_{i}(\kappa)\right\rangle_{i=1}^{m}: \kappa \in \aleph\right\}$, $A_{\aleph}^{(2)}=\left\{\left\langle\kappa,\left[\zeta_{i}^{-}(\kappa), \zeta_{i}^{+}(\kappa)\right], \zeta_{i}(\kappa)\right\rangle_{i=1}^{m}: \kappa \in \aleph\right\}$, and
$A_{\aleph}^{(3)}=\left\{\left\langle\kappa,\left[\theta_{i}^{-}(\kappa), \theta_{i}^{+}(\kappa)\right], \theta_{i}(\kappa)\right\rangle_{i=1}^{m}: \kappa \in \aleph\right\} \quad$ be the three CmPFSs on $\aleph$.

Proofs (1) and (2) are trivial.

(3) $A_{\aleph}^{(1)} \subseteq_{P} A_{\aleph}^{(2)}$ implies $\eta_{i}^{-}(\kappa) \leq \zeta_{i}^{-} \quad(\kappa), \eta_{i}^{+}(\kappa) \leq \zeta_{i}^{+}(\kappa)$ and $\eta_{i}(\kappa) \leq \zeta_{i}(\kappa)$.

Similarly $A_{\aleph}^{(2)} \subseteq_{P} A_{\aleph}^{(3)}$ implies $\zeta_{i}^{-}(\kappa) \leq \theta_{i}^{-}(\kappa), \zeta_{i}^{+}(\kappa) \leq \theta_{i}^{+}$ $(\kappa)$ and $\zeta_{i}(\kappa) \leq \theta_{i}(\kappa)$.

Combining the two facts, we obtain $\eta_{i}^{-}(\kappa) \leq \theta_{i}^{-}(\kappa), \eta_{i}^{+}(\kappa) \leq \theta_{i}^{+}(\kappa)$ and $\eta_{i}(\kappa) \leq \theta_{i}(\kappa)$ for all $i=1,2, \ldots, m$ and $\kappa \in \aleph$. This implies that $A_{\aleph}^{(1)} \subseteq_{P} A_{\aleph}^{(3)}$.

(4) Consider $\quad A_{\aleph}^{(1)} \cup_{P}\left(A_{\aleph}^{(2)} \cup_{P} A_{\aleph}^{(3)}\right) \quad=A_{\aleph}^{(1)} \cup_{P}(\{$ $\left\langle\kappa,\left[\zeta_{i}^{-}(\kappa) \vee \theta_{i}^{-}(\kappa), \zeta_{i}^{+}(\kappa) \vee \theta_{i}^{+}(\kappa)\right], \zeta_{i}(\kappa) \vee \theta_{i}(\kappa)\right\rangle_{i=1}^{\kappa}: \kappa \epsilon$ $\aleph\})=\left\{\left\langle\kappa,\left[\eta_{i}^{-}(\kappa) \vee\left(\zeta_{i}^{-}(\kappa) \vee \theta_{i}^{-}(\kappa)\right), \quad \eta_{i}^{+}(\kappa) \vee\left(\zeta_{i}^{+}\right.\right.\right.\right.$ $\left.\left.\left.(\kappa) \vee \theta_{i}^{+}(\kappa)\right)\right], \quad \quad \eta_{i}^{-}(\kappa) \vee\left(\zeta_{i}(\kappa) \vee \theta_{i}(\kappa)\right)\right\rangle$ $\left.m_{i=1}^{m}: \kappa \in \aleph\right\}=\{\langle\kappa$

$\left.\left.{ }^{-}(\kappa)\right) \vee \theta_{i}^{-}(\kappa),\left(\eta_{i}^{+}(\kappa) \vee \zeta_{i}^{+}(\kappa)\right) \vee \theta_{i}^{+}(\kappa)\right],\left(\eta_{i}^{-}\right.$

$\left.\left.\left.(\kappa) \vee \zeta_{i}(\kappa)\right) \vee \theta_{i}(\kappa)\right\rangle_{i=1}^{m}: \kappa \in \aleph\right\}=\left(A_{\aleph}^{(1)} \cup_{P} A_{\aleph}^{(2)}\right) \cup_{P} A_{\aleph}^{(3)}$.

(5) Similar to (4).

(6) $\quad A_{\aleph}^{(1)} \cup_{P}\left(A_{N}^{(2)} \cap_{P} A_{\aleph}^{(3)}\right)=A_{\aleph}^{(1)} \cup_{P}\left\{\left\langle\kappa,\left[\zeta_{i}^{-}(\kappa) \wedge \theta_{i}^{-}\right.\right.\right.$

$(\kappa)$, $\left.\left.\left.\zeta_{i}^{+}(\kappa) \wedge \theta_{i}^{+}(\kappa)\right], \zeta_{i}(\kappa) \wedge \theta_{i}(\kappa)\right\rangle_{i=1}^{m}: \kappa \in \aleph\right\}=\left\{\left\langle\kappa, \quad\left[\eta_{i}^{-}\right.\right.\right.$ $\left.(\kappa) \vee\left(\zeta_{i}^{-}(\kappa) \wedge \theta_{i}^{-}(\kappa)\right), \eta_{i}^{+}(\kappa) \vee\left(\zeta_{i}^{+}(\kappa) \wedge \theta_{i}^{+}(\kappa)\right)\right], \quad \eta_{i}(\kappa) \vee$ $\left.\left.\left(\zeta_{i}(\kappa) \wedge \theta_{i}(\kappa)\right)\right\rangle_{i=1}^{m}: \kappa \in \aleph\right\}=\left\{\left\langle\kappa,\left[\left(\eta_{i}^{-}(\kappa) \vee \zeta_{i}^{-}(\kappa)\right) \wedge\left(\eta_{i}^{-}\right.\right.\right.\right.$ $\left.\left.(\kappa) \vee \theta_{i}^{-}(\kappa)\right),\left(\eta_{i}^{+}(\kappa) \vee \zeta_{i}^{+}(\kappa)\right) \wedge\left(\eta_{i}^{+}(\kappa) \vee \theta_{i}^{+}(\kappa)\right)\right],\left(\eta_{i}(\kappa)\right.$ $\left.\vee \zeta_{i}(\kappa)\right) \wedge\left(\begin{array}{ll}\eta_{i} & \left.\left.(\kappa) \vee \theta_{i}(\kappa)\right)\right\rangle_{i=1}^{m}: \kappa \in \aleph\end{array}\right\}=\left(\begin{array}{ll}A_{\aleph}^{(1)} \cup_{P} & A_{\aleph}^{(2)}\end{array}\right)$ $\cap_{P}\left(A_{\aleph}^{(1)} \cup_{P} A_{\aleph}^{(3)}\right)$.

(7) Similar to (6)

Remark 1. In rest of the section, we will take $A_{\aleph}^{(1)}=$ $\left\{\left\langle\kappa,\left[\eta_{i}^{-}(\kappa), \eta_{i}^{+}(\kappa)\right], \eta_{i}(\kappa)\right\rangle_{i=1}^{m}: \kappa \in \aleph\right\}, \quad A_{\aleph}^{(2)}=\left\{\left\langle\kappa,\left[\zeta_{i}^{-}(\kappa)\right.\right.\right.$, $\left.\left.\left.\zeta_{i}^{+}(\kappa)\right], \zeta_{i}(\kappa)\right\rangle_{i=1}^{m}: \kappa \in \aleph\right\}$, and $A_{\aleph}^{(3)}=\left\{\left\langle\kappa,\left[\theta_{i}^{-}(\kappa), \theta_{i}^{+}(\kappa)\right]\right.\right.$, $\left.\left.\theta_{i}(\kappa)\right\rangle_{i=1}^{m}: \kappa \in \aleph\right\}$ as CmPFSs.

Proposition 1. Let $A_{\aleph}^{(1)}$ and $A_{\aleph}^{(2)}$. Then,

(1) $\left(A_{\aleph}^{(1)} \cup_{P} A_{\aleph}^{(2)}\right)^{c}=\left(A_{\aleph}^{(1)}\right)^{c} \cap_{P}\left(A_{\aleph}^{(2)}\right)^{c}$

(2) $\left(A_{\aleph}^{(1)} \cap_{P} A_{\aleph}^{(2)}\right)^{c}=\left(A_{\aleph}^{(1)}\right)^{c} \cup_{P}\left(A_{\aleph}^{(2)}\right)^{c}$

Proof. Let $\quad A_{\aleph}^{(1)}=\left\{\left\langle\kappa,\left[\eta_{i}^{-}(\kappa), \eta_{i}^{+}(\kappa)\right] \quad, \eta_{i}(\kappa)\right\rangle_{i=1}^{m}: \kappa \in\right.$ $\aleph\}$ and $A_{\aleph}^{(2)}=\left\{\left\langle\kappa,\left[\zeta_{i}^{-}(\kappa), \zeta_{i}^{+}(\kappa)\right], \zeta_{i}(\kappa)\right\rangle_{i=1}^{m}: \kappa \in \aleph\right\}$.

(1) $\left(A_{\aleph}^{(1)} \cup{ }_{P} A_{\aleph}^{(2)}\right)^{c}=\left(\left\{\left\langle\kappa,\left[\eta_{i}^{-}(\kappa) \vee \zeta_{i}^{-}(\kappa), \eta_{i}^{+}(\kappa) \vee \zeta_{i}^{+}(\kappa)\right], \eta_{i}(\kappa) \vee \zeta_{i}(\kappa)\right\rangle_{i=1}^{m}\right.\right.$ : $\kappa \in \aleph\})^{c}=\left\{\left\langle\kappa,\left[1-\left(\eta_{i}^{-}(\kappa) \vee \zeta_{i}^{-}(\kappa)\right), 1-\left(\eta_{i}^{+}(\kappa) \vee \zeta_{i}^{+}\right.\right.\right.\right.$ $\left.\left.(\kappa))], 1-\left(\eta_{i}(\kappa) \vee \zeta_{i}(\kappa)\right)\right\rangle_{i=1}^{m}: \kappa \in \aleph\right\}=\left\{\left\langle\kappa,\left[\left(1-\eta_{i}^{-}\right.\right.\right.\right.$ $\left.(\kappa)) \wedge\left(1-\zeta_{i}^{-}(\kappa)\right),\left(1-\eta_{i}^{+}(\kappa)\right) \wedge\left(1-\zeta_{i}^{+}(\kappa)\right)\right],\left(1-\eta_{i}\right.$ $\left.\left.(\kappa)) \wedge\left(1-\zeta_{i}(\kappa)\right)\right\rangle_{i=1}^{m}: \kappa \in \aleph\right\}=\left(A_{\aleph}^{(1)}\right)^{c} \cap_{P}\left(A_{\aleph}^{(2)}\right)^{c}$.

(2) Similar to (1).

Definition 5. Let $A_{\aleph}=\left\{\left\langle\kappa,\left[\eta_{i}^{-}(\kappa), \eta_{i}^{+}(\kappa)\right], \eta_{i}(\kappa)\right\rangle_{i=1}^{m}: \kappa \in \aleph\right\}$ be a CmPFS with discourse $\aleph$. Then, the support of $A_{\aleph}$ is the collection of all those crisp elements from $\aleph$ which contribute to its cubic $m$-polar behaviour. That is, 


$$
\operatorname{Supp}\left(A_{\aleph}\right)=\left\{\kappa \in \aleph:\left[\eta_{i}^{-}(\kappa), \eta_{i}^{+}(\kappa)\right] \neq \mathbf{0}_{\mathfrak{m}} \text { or } \eta_{i}(\kappa) \neq 1 \text { for at least one } i\right\}
$$

where $\mathbf{0}_{\mathfrak{m}}$ is the null CmPFN.

Example 1. Let $\aleph=\left\{\kappa_{1}, \kappa_{2}, \kappa_{3}, \kappa_{4}\right\}$ be the universe, then a CmPFS $A_{\aleph}$ on $\aleph$ is given by

$$
\begin{array}{r}
A_{\aleph}=\left\{\left(\kappa_{1},\langle[0.1,0.2],[0.8,0.9],[0,0], 0.9,1,0.3\rangle\right)\right. \\
\left(\kappa_{2},\langle[0,0],[0,0],[0,0], 1,1,1\rangle\right) \\
\left(\kappa_{3},\langle[0,0],[0,0],[0,0.7], 1,1,0.5\rangle\right) \\
\left.\left(\kappa_{4},\langle[1,1],[1,1],[1,1], 0,0,0\rangle\right)\right\} .
\end{array}
$$

The CMPFS $A_{\aleph}$ can be expressed in the tabular form as given by Table 1 . Then, $\operatorname{Supp}\left(A_{\aleph}\right)=\left\{\kappa_{1}, \kappa_{3}, \kappa_{4}\right\}$.

Definition 6. The core of a CmPFS $A_{\aleph}=\left\{\left\langle\kappa,\left[\eta_{i}^{-}(\kappa), \eta_{i}^{+}(\kappa)\right], \eta_{i}(\kappa)\right\rangle_{i=1}^{m}: \kappa \in \aleph\right\}$ is the collection of crisp elements $\kappa$ from $\aleph$ for which $\left[\eta_{i}^{-}(\kappa), \eta_{i}^{+}(\kappa)\right]=\mathbf{1}_{\mathfrak{m}}$ and $\eta_{i}(\kappa)=\mathbf{0}_{\mathfrak{m}}$ for all $i$. Mathematically,

$$
\operatorname{Cor}\left(A_{\aleph}\right)=\left\{\kappa \in \aleph:\left[\eta_{i}^{-}(\kappa), \eta_{i}^{+}(\kappa)\right]=\mathbf{1}_{\mathfrak{m}} \text { and } \eta_{i}(\kappa)=\mathbf{0}_{\mathfrak{m}} \text { for all } i\right\},
$$

where $\mathbf{1}_{\mathfrak{m}}$ is the absolute CmPFN.

Example 2. The core of the CmPFS in Example 1 is given by

$$
\operatorname{Cor}\left(A_{\aleph}\right)=\left\{\kappa_{4}\right\} \text {. }
$$

Definition 7. The height of a CmPFS is the maximum membership value that a certain alternative can attain. If $A_{\aleph}=\left\{\left\langle\kappa,\left[\eta_{i}^{-}(\kappa), \eta_{i}^{+}(\kappa)\right], \eta_{i}(\kappa)\right\rangle_{i=1}^{m}: \kappa \in \aleph\right\} \quad$ is a CmPFS, then the height is given by

$$
\underline{h t}\left(A_{\aleph}\right)=\max _{\{\kappa \in \aleph, i\}} \eta_{i}^{+}(\kappa) \text {. }
$$

If the height of a CmPFS finds its value to be 1 , then it is said to be a normal CmPFS:

$$
A_{\aleph} \text { is normal } \Leftrightarrow \underline{h t}\left(A_{\aleph}\right)=1 .
$$

Example 3. The CmPFS given in Example 1 can be referred as a normal one because its height is 1 , that is, $\underline{h t}\left(A_{\aleph}\right)=1$.

Remark 2. If $A_{\aleph}^{(1)}$ and $A_{\aleph}^{(2)}$ are CmPFSs over a crisp domain $\aleph$, then

$$
\begin{aligned}
& A_{\aleph}^{(1)} \subseteq_{P} A_{\aleph}^{(2)} \Rightarrow \underline{h t}\left(A_{\aleph}^{(1)}\right) \leq \underline{h t}\left(A_{\aleph}^{(2)}\right), \\
& A_{\aleph}^{(1)} \subseteq_{R} A_{\aleph}^{(2)} \Rightarrow \underline{h t}\left(A_{\aleph}^{(1)}\right) \leq \underline{h t}\left(A_{\aleph}^{(2)}\right) .
\end{aligned}
$$

However, the converse does not hold in general. The counter example is given as follows.

Example 4. Consider the two CmPFSs, namely, $A_{\aleph}^{(1)}$ and $A_{\aleph}^{(2)}$ as given by

$$
\begin{aligned}
A_{\aleph}^{(1)}=\{ & \left(\kappa_{1},\langle[0.10,0.12],[0.30,0.36],[0.26,0.40], 0.71,0.68,0.90\rangle\right) \\
& \left(\kappa_{2},\langle[0.40,0.47],[0.50,0.60],[0.47,0.57], 0.80,0.80,0.76\rangle\right) \\
& \left(\kappa_{3},\langle[0.50,0.55],[0.60,0.66],[0.23,0.34], 0.56,0.69,0.44\rangle\right) \\
& \left.\left(\kappa_{4},\langle[0.89,0.93],[0.10,0.36],[0.56,0.60], 0.70,0.20,0.47\rangle\right)\right\}, \\
A_{\aleph}^{(2)}=\{ & \left(\kappa_{1},\langle[0.88,0.10],[0.64,0.70],[0.60,0.74], 0.29,0.32,0.10\rangle\right) \\
& \left(\kappa_{2},\langle[0.53,0.60],[0.40,0.50],[0.43,0.53], 0.20,0.20,0.24\rangle\right) \\
& \left(\kappa_{3},\langle[0.45,0.50],[0.34,0.40],[0.66,0.77], 0.44,0.31,0.56\rangle\right) \\
& \left.\left(\kappa_{4},\langle[0.07,0.11],[0.64,0.90],[0.40,0.44], 0.30,0.80,0.53\rangle\right)\right\} .
\end{aligned}
$$

$\underset{A_{\aleph}^{(1)} \Phi_{P, R} A_{\aleph}^{(2)} .}{\text { Clearly }} \underline{h t}\left(A_{\aleph}^{(1)}\right)=0.93 \leq 1=\underline{h t}\left(A_{\aleph}^{(2)}\right) . \quad$ However,

Theorem 2. For a CmPFS $A_{\aleph}$, following results are straightforward:

(1) $A_{\aleph}=A_{\aleph} \cup_{P} A_{\aleph}, A_{\aleph} \cap_{P} A_{\aleph}=A_{\aleph}$

(2) $A_{\aleph}=A_{\aleph} \cup_{R} A_{\aleph}, A_{\aleph} \cap_{R} A_{\aleph}=A_{\aleph}$

(3) $A_{\aleph} \oplus_{P} A_{\aleph}=A_{\aleph} \Leftrightarrow A_{\aleph}=\mathbf{1}_{\mathfrak{m}}$ or $\mathbf{1}_{\mathfrak{m}}$

(4) $A_{\aleph} \otimes_{P} A_{\aleph}=A_{\aleph} \Leftrightarrow A_{\aleph}=\mathbf{0}_{\mathfrak{m}}$ or $\mathbf{1}_{\mathfrak{m}}$

(5) $A_{\aleph} \oplus_{R} A_{\aleph}=A_{\aleph} \Leftrightarrow A_{\aleph}=\mathbf{0}_{\mathfrak{m}}$ or $\mathbf{1}_{\mathfrak{m}}$

(6) $A_{\aleph} \otimes_{R} A_{\aleph}=A_{\aleph} \Leftrightarrow A_{\aleph}=\mathbf{0}_{\mathfrak{m}}$ or $\mathbf{1}_{\mathfrak{m}}$
(7) $\varsigma A_{\aleph}=A_{\aleph}$ and $\left(A_{\aleph}\right)^{\varsigma}=A_{\aleph} \Leftrightarrow \varsigma=1$

Theorem 3. Let $A_{\aleph}, B_{\aleph}, A_{\aleph}^{(1)}$, and $A_{\aleph}^{(2)}$ be the CmPFS with usual notations. Then,

(1) If $A_{\aleph} \subseteq_{P} A_{\aleph}^{(1)}$ and $A_{\aleph} \subseteq_{P} A_{\aleph}^{(2)}$, then $A_{\aleph} \subseteq_{P} A_{\aleph}^{(1)} \cap{ }_{p} A_{\aleph}^{(2)}$

(2) If $A_{\aleph}^{(1)} \subseteq_{P} A_{\aleph}$ and $A_{\aleph}^{(2)} \subseteq_{P} A_{\aleph}$, then $A_{\aleph}^{(1)} \cup_{p} A_{\aleph}^{(2)} \subseteq_{P} A_{\aleph}$

(3) If $\quad A_{\aleph}^{(1)} \subseteq_{P} A_{\aleph}$ and $A_{\aleph} \quad(2) \subseteq_{P}{ }^{\aleph} B$, then $\quad A_{\aleph}^{(1)}$ $\cup_{p} A_{\aleph}^{(2)} \subseteq_{P} A_{\aleph} \cup_{P} B_{\aleph}$ and $A_{\aleph}^{(1)} \cap_{p} A_{\aleph}^{(2)} \subseteq_{P} A_{\aleph} \cap_{p} B_{\aleph}$

Theorem 4. Let $A_{\aleph}, A_{\aleph}^{(1)}$, and $A_{\aleph}^{(2)}$ be the usual CmPFS. Then, 
TABle 1: CmPFS $A_{\aleph}$.

\begin{tabular}{cc}
\hline$\kappa_{1}$ & $\langle[0.1,0.2],[0.8,0.9],[0,0], 0.9,1,0.3\rangle$ \\
$\kappa_{2}$ & $\langle[0,0],[0,0],[0,0], 1,1,1\rangle$ \\
$\kappa_{3}$ & $\langle[0,0],[0,0],[0,0.7], 1,1,0.5\rangle$ \\
$\kappa_{4}$ & $\langle[1,1],[1,1],[1,1], 0,0,0\rangle$ \\
\hline
\end{tabular}

(1) $\left(\left(A_{\aleph}\right)^{c}\right)^{c}=A_{\aleph}$

(2) $A_{\aleph}^{(1)} \subseteq_{P} A_{\aleph}^{(2)} \Leftrightarrow\left(A_{\aleph}^{(2)}\right)^{c} \subseteq_{P}\left(A_{\aleph}^{(1)}\right)^{c}$

(3) $A_{\aleph}^{(1)}=A_{\aleph}^{(2)} \Leftrightarrow\left(A_{\aleph}^{(1)}\right)^{c}=\left(A_{\aleph}^{(2)}\right)^{c}$

(4) $\left(A_{\aleph}^{(1)} \oplus_{P} A_{\aleph}^{(2)}\right)^{c}=\left(A_{\aleph}^{(1)}\right)^{c} \otimes_{P}\left(A_{\aleph}^{(2)}\right)^{c}$

(5) $\left(A_{\aleph}^{(1)} \oplus_{P} A_{\aleph}^{(2)}\right)^{c}=\left(A_{\aleph}^{(1)}\right)^{c} \otimes_{P}\left(A_{\aleph}^{(2)}\right)^{c}$

(6) $\left(\left(A_{\aleph}\right)^{\varsigma}\right)^{c}=\varsigma\left(\left(A_{\aleph}\right)^{c}\right)$

(7) $\left(\varsigma\left(A_{\aleph}\right)^{c}\right)^{c}=\left(A_{\aleph}\right)^{\varsigma}$

(8) $\left(\varsigma A_{\aleph}\right)^{c}=\left(\left(A_{\aleph}\right)^{c}\right)^{\varsigma}$

Proof. (1) and (3) are straightforward.

$$
A_{\aleph}^{(1)} \subseteq_{P} A_{\aleph}^{(2)}
$$

(2) Let $\Leftrightarrow \eta_{i}^{-}(\kappa) \leq \zeta_{i}^{-}(\kappa), \eta_{i}^{+}(\kappa) \leq \zeta_{i}^{+}(\kappa)$ and $\eta_{i}(\kappa) \leq \zeta_{i}(\kappa)$

$\Leftrightarrow 1-\zeta_{i}^{-}(\kappa) \leq 1-\eta_{i}^{-}(\kappa), 1-\zeta_{i}^{+}(\kappa) \leq 1-\eta_{i}^{+}(\kappa)$ and $1-\zeta_{i}(\kappa) \leq 1-\eta_{i}(\kappa)$ $\Leftrightarrow\left(A_{\aleph}^{(2)}\right)^{c} \subseteq_{P}\left(A_{\aleph}^{(1)}\right)^{1}$.

$\left(A_{R}^{(1)} \oplus_{P} A_{R}^{(2)}\right)^{c}$

$=\left\{\left\langle\kappa,\left[\eta_{i}^{-}(\kappa)+\zeta_{i}(\kappa)-\eta_{i}^{-}(\kappa) \zeta \zeta_{i}^{-}(\kappa), \eta_{i}^{+}(\kappa)+\zeta_{i}^{+}(\kappa)-\eta_{i}^{+}(\kappa) \zeta_{i}^{+}(\kappa)\right]\right.\right.$,

$\left.\left.\eta_{i}(\kappa)+\zeta_{i}(\kappa)-\eta_{i}(\kappa) \zeta_{i}(\kappa)\right\rangle_{i=1}^{m}: \kappa \in \mathbb{N}\right\}^{c}$

(4) $=\left\{\left\langle\kappa,\left[1-\eta_{i}^{+}(\kappa)-\zeta_{i}^{+}(\kappa)+\eta_{i}^{+}(\kappa) \zeta_{i}^{+}(\kappa), 1-\eta_{i}^{-}(\kappa)-\zeta_{i}(\kappa)+\eta_{i}^{-}(\kappa) \zeta_{i}(\kappa)\right]\right.\right.$,

$\left.\left.1-\eta_{i}(\kappa)-\zeta_{i}(\kappa)+\eta_{i}(\kappa) \zeta_{i}(\kappa)\right\rangle_{i=1}^{m}: \kappa \in \mathbb{N}\right\}^{c}$

$=\left\{\left\langle\kappa,\left[1-\eta_{i}^{+}(\kappa), 1-\eta_{i}^{-}(\kappa)\right], 1-\eta_{i}(\kappa)\right\rangle_{i=1}^{m}: \kappa \in \mathbb{N}\right\} \otimes_{P}\left\{\left\langle\kappa,\left[1-\zeta_{i}^{\dagger}(\kappa), 1-\zeta_{i}(\kappa)\right], 1-\zeta_{i}(\kappa)\right\rangle_{i=1}^{m}: \kappa \in \mathbb{N}\right\}$ $=\left(A_{\aleph}^{(1)}\right)^{c} \otimes_{p}\left(A_{\aleph}^{(2)}\right)^{c}$.

$\left(A_{\mathrm{R}}^{(1)} \otimes_{p} A_{\mathrm{R}}^{(2)}\right)^{c}$

$=\left(\left\{\left\langle\kappa,\left[\eta_{i}^{-}(\kappa) \zeta_{i}(\kappa), \eta_{i}^{+}(\kappa) \zeta_{i}^{+}(\kappa)\right], \eta_{i}(\kappa) \zeta_{i}(\kappa)\right\rangle_{i=1}^{m}: \kappa \in \mathcal{N}\right\}\right)^{c}$

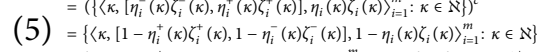

$=\left\{\left\langle\kappa,\left[1-\eta_{i}^{+}(\kappa), 1-\eta_{i}^{-}(\kappa)\right], 1-\eta_{i}(\kappa)\right\rangle_{i=1}^{m}: \kappa \in \mathbb{N}\right\} \oplus_{p}\left\{\left\langle\left\langle,\left[1-\zeta_{i}^{+}(\kappa), 1-\zeta_{i}^{-}(\kappa)\right], 1-\zeta_{i}(\kappa)\right\rangle_{i=1}^{m}: \kappa \in \mathbb{N}\right\}\right.$ $=\left(A_{x}^{(1)}\right)^{c} \oplus_{p}\left(A_{x}^{(2)}\right)^{c}$.

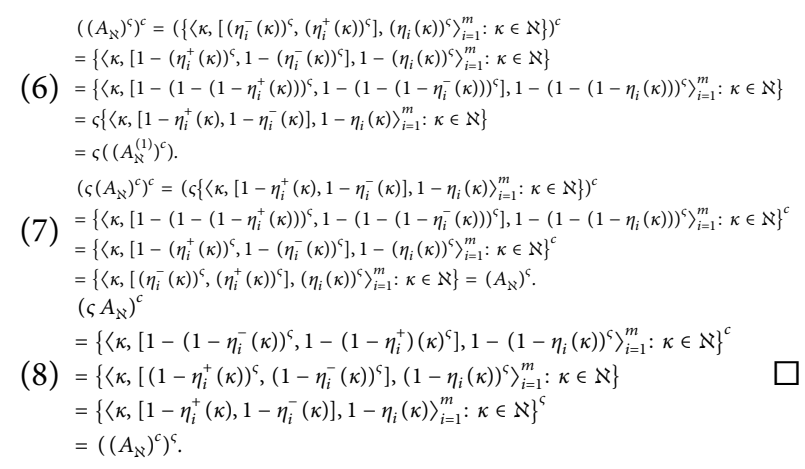

Theorem 5. The two famous laws of crisp set theory, law of contradiction and excluded middle, do not hold in CmPF P-environment as well as R-environment. That is,

(1) $A_{\aleph} \cap_{P}\left(A_{\aleph}\right)^{c} \neq \mathbf{0}_{\mathfrak{m}}$

(2) $A_{\aleph} \cup_{P}\left(A_{\aleph}\right)^{c} \neq \mathbf{1}_{\mathfrak{m}}$

(3) $A_{\aleph} \cap_{R}\left(A_{\aleph}\right)^{c} \neq \mathbf{1}_{\mathfrak{m}}$

(4) $A_{\aleph} \cup_{R}\left(A_{\aleph}\right)^{c} \neq \mathbf{1}_{\mathfrak{m}}$

The counter example for CmPFSs is given as follows.

Example 5. Let

$$
A_{\aleph}=\kappa_{2} \kappa_{1}\left[\begin{array}{c}
{[0.10,0.12],[0.30,0.36],[0.26,0.40], 0.71,0.68,0.90} \\
\kappa_{3} \\
\kappa_{4}
\end{array}\left[\begin{array}{c}
0.40,0.47],[0.50,0.60],[0.47,0.57], 0.80,0.80,0.76 \\
{[0.89,0.93],[0.10,0.36],[0.56,0.60], 0.70,0.20,0.47}
\end{array}\right]\right.
$$

be a CmPFS. Its complement is given by

$$
\left(A_{\aleph}\right)^{c}=\begin{array}{r}
\kappa_{1} \\
\kappa_{2}
\end{array} \quad \begin{gathered}
{[0.88,0.90],[0.64,0.70],[0.60,0.74], 0.29,0.32,0.10} \\
\kappa_{3} \\
\kappa_{4}
\end{gathered}\left[\begin{array}{l}
0.43,0.60],[0.40,0.50],[0.43,0.53], 0.20,0.20,0.24 \\
{[0.07,0.11],[0.64,0.90],[0.40,0.44], 0.30,0.80,0.53}
\end{array}\right]
$$

Then,

$$
\begin{aligned}
& A_{\aleph} \underset{P}{\cap}\left(A_{\aleph}\right)^{c}=\kappa_{1} \kappa_{\kappa_{3}}\left[\begin{array}{c}
{[0.10,0.12],[0.30,0.36],[0.26,0.40], 0.29,0.32,0.10} \\
{[0.40,0.47],[0.40,0.50],[0.43,0.53], 0.20,0.20,0.24} \\
{[0.45,0.50],[0.34,0.40],[0.23,0.34], 0.44,0.31,0.44} \\
\kappa_{4}
\end{array}\right] \neq \mathbf{0}_{\mathfrak{m}}, \\
& A_{\aleph} \bigcap_{R}\left(A_{\aleph}\right)^{c}=\kappa_{1} \kappa_{2}\left[\begin{array}{c}
{[0.10,0.12],[0.30,0.36],[0.26,0.40], 0.71,0.68,0.90} \\
\kappa_{3} \\
\kappa_{4}
\end{array}\left[\begin{array}{c}
0.40,0.47],[0.40,0.50],[0.43,0.53], 0.80,0.80,0.76 \\
{[0.07,0.11],[0.10,0.36],[0.40,0.44], 0.70,0.80,0.53}
\end{array}\right] \neq \mathbf{0}_{\mathfrak{m}} .\right.
\end{aligned}
$$

Similarly, other cases can be addressed. 
Theorem 6. Let $A_{\aleph}, A_{\aleph}^{(1)}$, and $A_{\aleph}^{(2)}$ be the usual CmPFS. Then,

(1) $A_{\aleph}^{(1)} \subseteq_{P} A_{\aleph}^{(2)} \Leftrightarrow \varsigma\left(A_{\aleph}^{(1)}\right) \subseteq_{P} \varsigma\left(A_{\aleph}^{(2)}\right)$

(2) $A_{\aleph}^{(1)}=A_{\aleph}^{(2)} \Leftrightarrow \varsigma\left(A_{\aleph}^{(1)}\right)=\varsigma\left(A_{\aleph}^{(2)}\right)$

(3) $\varsigma\left(A_{\aleph}^{(1)} \oplus_{P} A_{\aleph}^{(2)}\right)=\left(\left(\left(A_{\aleph}^{(1)} \oplus_{P} A_{\aleph}^{(2)}\right)^{c}\right)^{\varsigma}\right)^{c}$

(4) $\varsigma\left(A_{\aleph}^{(1)} \otimes_{P} A_{\aleph}^{(2)}\right)=\left(\left(\left(A_{\aleph}^{(1)} \otimes_{P} A_{\aleph}^{(2)}\right)^{c}\right)^{\varsigma}\right)^{c}$

(5) $\left(A_{\aleph}^{(1)} \otimes_{P} A_{\aleph}^{(2)}\right)^{\varsigma}=\left(A_{\aleph}^{(1)}\right)^{\varsigma} \otimes_{P}\left(A_{\aleph}^{(2)}\right)^{\varsigma}$

(6) $\varsigma\left(A_{\aleph}^{(1)}\right) \otimes_{P} \varsigma\left(A_{\aleph}^{(2)}\right)=\left(\left(\left(A_{\aleph}^{(1)}\right)^{c}\right)^{\varsigma} \oplus_{P}\left(\left(A_{\aleph}^{(2)}\right)^{c}\right)^{\varsigma}\right)^{c}$

(7) $\varsigma\left(A_{\aleph}^{(1)} \oplus_{P} A_{\aleph}^{(2)}\right)=\varsigma\left(A_{\aleph}^{(1)}\right) \oplus_{P} \varsigma\left(A_{\aleph}^{(2)}\right)$

Proof. We discuss proof for (1), (3), (6), and (7). Rest of the parts have similar proof.

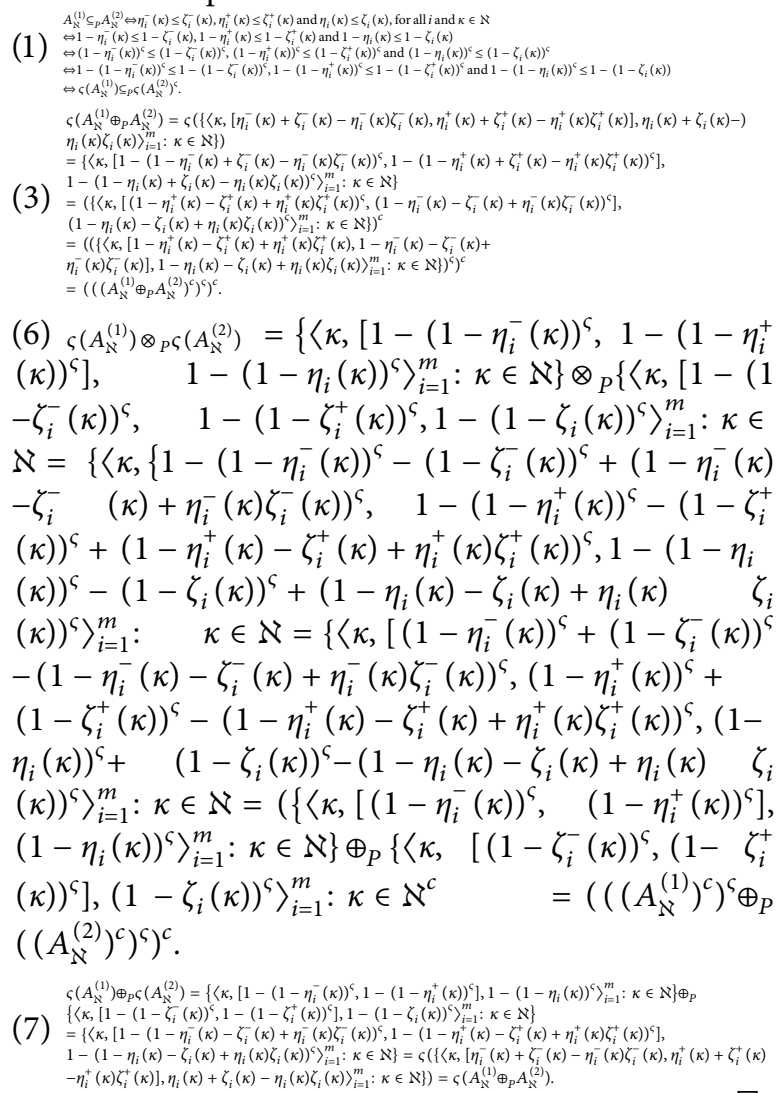

Definition 8. Let $g, h \in[0,1]$. The upper g-level set of a CmPFS,

$$
A_{\aleph}=\left\{\left\langle\kappa,\left[\eta_{i}^{-}(\kappa), \eta_{i}^{+}(\kappa)\right], \eta_{i}(\kappa)\right\rangle_{i=1}^{m}: \kappa \in \aleph\right\},
$$

is denoted by $\mathfrak{U}\left(A_{\aleph}, g\right)$ and is defined as

$$
\mathfrak{U}\left(A_{\aleph}, g\right)=\left\{\kappa \in \aleph: \eta_{i}^{-}(\kappa) \geq g\right\} .
$$

The lower h-level set of the set $A_{\aleph}$ is denoted by $\mathfrak{Q}\left(A_{\aleph}, h\right)$ and is defined as

$$
\mathfrak{L}\left(A_{\aleph}, h\right)=\left\{\kappa \in \aleph: \eta_{i}(\kappa) \leq h\right\} .
$$

The strong upper g-level set and the strong lower h-level set of $A_{\aleph}$ can be, respectively, defined as

$$
\begin{aligned}
\mathfrak{U}_{s}\left(A_{\aleph}, g\right) & =\left\{\kappa \in \aleph: \eta_{i}^{-}(\kappa)>g\right\}, \\
\mathfrak{Q}_{s}\left(A_{\aleph}, h\right) & =\left\{\kappa \in \aleph: \eta_{i}(\kappa)<h\right\} .
\end{aligned}
$$

Moreover, if we put the restriction $g+h \leq 1$, then we can define $(\mathrm{g}, \mathrm{h})$-level set of $A_{\aleph}$ as

$$
{ }^{(g, h)}\left(A_{\aleph}\right)=\left\{\kappa \in \aleph: \eta_{i}^{-}(\kappa) \geq g, \eta_{i}(\kappa) \leq h\right\} .
$$

Similarly, strong $(\mathrm{g}, \mathrm{h})$-level set of $A_{\aleph}$ can be defined as

$$
{ }^{(g, h)}\left(A_{\aleph}\right)_{s}=\left\{\kappa \in \aleph: \eta_{i}^{-}(\kappa)>g, \eta_{i}(\kappa)<h\right\} .
$$

Example 6. In Example 4, the level sets are given as follows:

$$
\begin{aligned}
\mathfrak{U}\left(A_{\aleph}, 0.23\right) & =\left\{\kappa_{2}, \kappa_{3}\right\} \cdot \mathfrak{U}_{s}\left(A_{\aleph}, 0.23\right)=\left\{\kappa_{2}\right\}, \\
\mathfrak{L}\left(A_{\aleph}, 0.8\right) & =\left\{\kappa_{2}, \kappa_{3}, \kappa_{4}\right\} \cdot \mathfrak{Q}_{s}\left(A_{\aleph}, 0.68\right)=\left\{\kappa_{3}, \kappa_{4}\right\}, \\
{ }^{(0.23,0.8)}\left(A_{\aleph}\right) & =\left\{\kappa_{2}, \kappa_{3}\right\} \cdot{ }^{(0.23,0.8)}\left(A_{\aleph}\right)_{s}=\{\} .
\end{aligned}
$$

Remark 3. ${ }^{(g, h)}\left(A_{\aleph}\right)=\left\{\kappa \in \aleph: \eta_{i}^{-}(\kappa) \geq g, \eta_{i}(\kappa) \leq h\{\kappa \in \aleph:\right.$ $\left.\eta_{i}^{-}(\kappa) \geq g\right\} \cap\left\{\kappa \in \mathcal{K}: \eta_{i}(\kappa) \leq h\right\}=\mathfrak{U}\left(A_{\aleph}, g\right) \cap \mathfrak{Q}\left(A_{\aleph}, h\right)$.

This shows that $(\mathrm{g}, \mathrm{h})$-level set of $A_{\aleph}$ is actually an intersection of upper g-level and lower h-level sets.

Definition 9. Two CmPFSs are given by

$$
\begin{aligned}
& A_{\aleph}^{(1)}=\left\{\left\langle\kappa_{1},\left[\eta_{i}^{-}\left(\kappa_{1}\right), \eta_{i}^{+}\left(\kappa_{1}\right)\right], \eta_{i}\left(\kappa_{1}\right)\right\rangle_{i=1}^{m}: \kappa_{1} \in \aleph_{1}\right\}, \\
& A_{\aleph}^{(2)}=\left\{\left\langle\kappa_{2},\left[\zeta_{i}^{-}\left(\kappa_{2}\right), \zeta_{i}^{+}\left(\kappa_{2}\right)\right], \zeta_{i}\left(\kappa_{2}\right)\right\rangle_{i=1}^{m}: \kappa_{2} \in \aleph_{2}\right\} .
\end{aligned}
$$

The four versions of Cartesian products of the two CmPFSs are given by

$$
\begin{aligned}
& A_{\aleph}^{(1)} \times_{P} A_{\aleph}^{(2)}=\left\{\left\langle\left(\kappa_{1}, \kappa_{2}\right),\left[\eta_{i}^{-}\left(\kappa_{1}\right) \zeta_{i}^{-}\left(\kappa_{2}\right), \eta_{i}^{+}\left(\kappa_{1}\right) \zeta_{i}^{+}\left(\kappa_{2}\right)\right], \eta_{i}\left(\kappa_{1}\right) \zeta_{i}\left(\kappa_{2}\right)\right\rangle_{i=1}^{m}: \kappa_{2} \in \aleph_{2}, \kappa_{1} \in \aleph_{1}\right\}, \\
& A_{\aleph}^{(1)} \times_{R} A_{\aleph}^{(2)}=\left\{\left\langle\left(\kappa_{1}, \kappa_{2}\right),\left[\eta_{i}^{-}\left(\kappa_{1}\right) \zeta_{i}^{-}\left(\kappa_{2}\right), \eta_{i}^{+}\left(\kappa_{1}\right) \zeta_{i}^{+}\left(\kappa_{2}\right)\right], \eta_{i}\left(\kappa_{1}\right)+\zeta_{i}\left(\kappa_{2}\right)-\eta_{i}\left(\kappa_{1}\right) \zeta_{i}\left(\kappa_{2}\right)\right\rangle_{i=1}^{m}: \kappa_{2} \in \aleph_{2}, \kappa_{1} \in \aleph_{1}\right\}, \\
& A_{\aleph}^{(1)} \times_{P} A_{\aleph}^{(2)}=\left\{\left\langle\left(\kappa_{1}, \kappa_{2}\right),\left[\eta_{i}^{-}\left(\kappa_{1}\right) \wedge \zeta_{i}^{-}\left(\kappa_{2}\right), \eta_{i}^{+}\left(\kappa_{1}\right) \wedge \zeta_{i}^{+}\left(\kappa_{2}\right)\right], \eta_{i}\left(\kappa_{1}\right) \wedge \zeta_{i}\left(\kappa_{2}\right)\right\rangle_{i=1}^{m}: \kappa_{2} \in \aleph_{2}, \kappa_{1} \in \aleph_{1}\right\}, \\
& A_{\aleph}^{(1)} \times_{R} A_{\aleph}^{(2)}=\left\{\left\langle\left(\kappa_{1}, \kappa_{2}\right),\left[\eta_{i}^{-}\left(\kappa_{1}\right) \wedge \zeta_{i}^{-}\left(\kappa_{2}\right), \eta_{i}^{+}\left(\kappa_{1}\right) \wedge \zeta_{i}^{+}\left(\kappa_{2}\right)\right], \eta_{i}\left(\kappa_{1}\right) \vee \zeta_{i}\left(\kappa_{2}\right)\right\rangle_{i=1}^{m}: \kappa_{2} \in \aleph_{2}, \kappa_{1} \in \aleph_{1}\right\} .
\end{aligned}
$$

Interestingly, the cubic Cartesian product of the CmPFSs is again a CMPFS. 
Theorem 7. Let $A_{\aleph}^{(1)}, A_{\aleph}^{(2)}$, and $A_{\aleph}^{3}$ be CmPFSs. Then,

(1) $A_{\aleph}^{(1)} \cup_{R} A_{\aleph}^{(2)}=A_{\aleph}^{(2)} \cup_{R} A_{\aleph}^{(1)}$

(2) $A_{\aleph}^{(1)} \cap_{R} A_{\aleph}^{(2)}=A_{\aleph}^{(2)} \cap_{R} A_{\aleph}^{(1)}$

(3) $A_{\aleph}^{(1)} \subseteq_{R} A_{\aleph}^{(2)}$ and $A_{\aleph}^{(2)} \subseteq_{R} A_{\aleph}^{3}$ implies $A_{\aleph}^{(1)} \subseteq_{R} A_{\aleph}^{3}$

(4) $A_{\aleph}^{(1)} \cup_{R}\left(A_{\aleph}^{(2)} \cup_{R} A_{\aleph}^{3}\right)=\left(A_{\aleph}^{(1)} \cup_{R} A_{\aleph}^{(2)}\right) \cup_{R} A_{\aleph}^{3}$

(5) $A_{\aleph}^{(1)} \cap_{R}\left(A_{\aleph}^{(2)} \cap_{R} A_{\aleph} 63\right)=\left(A_{\aleph}^{(1)} \cap_{R} A_{\aleph}^{(2)}\right) \cap_{R} A_{\aleph}^{3}$

(6) $A_{\aleph}^{(1)} \cup_{R}\left(A_{\aleph}^{(2)} \cap_{R} A_{\aleph}^{3}\right)=\left(A_{\aleph}^{(1)} \cup_{R} A_{\aleph}^{(2)}\right) \cap_{R}$ $\left(A_{\aleph}^{(1)} \cup{ }_{R} A_{\aleph}^{3}\right)$

(7) $A_{\aleph}^{(1)} \cap_{R}\left(A_{\aleph}^{(2)} \cup_{R} A_{\aleph}^{3}\right)=\left(A_{\aleph}^{(1)} \cap_{R} A_{\aleph}^{(2)}\right) \cup_{R}$ $\left(A_{\aleph}^{(1)} \cap_{R} A_{\aleph}^{3}\right)$

Theorem 8. let $A_{\aleph}, \Phi_{\mathfrak{m}}$, and $\mathfrak{U}_{\mathfrak{m}}$ be the CmPFS, and null and absolute CmPFSs, respectively. Then,

(1) $A_{\aleph} \cup_{R} \Phi_{\mathfrak{m}}=A_{\aleph}$

(2) $A_{\aleph} \cap_{R} \Phi_{\mathfrak{m}}=\Phi_{\mathfrak{m}}$

(3) $A_{\aleph}=A_{\aleph} \cup_{R} A_{\aleph}$

(4) $A_{\aleph} \cap{ }_{R} A_{\aleph}=A_{\aleph}$

(5) $A_{\aleph} \cup_{R} \mathcal{U}_{\mathfrak{m}}=\mathfrak{U}_{\mathfrak{m}}$

(6) $A_{\aleph} \cap_{R} \mathfrak{U}_{\mathfrak{m}}=A_{\aleph}$

Proposition 2. Let $A_{\aleph}^{(1)}$ and $A_{\aleph}^{(2)}$. Then,

(1) $\left(A_{\aleph}^{(1)} \cup_{R} A_{\aleph}^{(2)}\right)^{c}=\left(A_{\aleph}^{(1)}\right)^{c} \cap_{R}\left(A_{\aleph}^{(2)}\right)^{c}$

(2) $\left(A_{\aleph}^{(1)} \cap_{R} A_{\aleph}^{(2)}\right)^{c}=\left(A_{\aleph}^{(1)}\right)^{c} \cup_{R}\left(A_{\aleph}^{(2)}\right)^{c}$

Theorem 9. Let $A_{\aleph}, B_{\aleph}, A_{\aleph}^{(1)}$, and $A_{\aleph}^{(2)}$ be the CmPFS with usual notations. Then,

(1) If $A_{\aleph}^{(1)} \subseteq_{R} A_{\aleph}^{(2)}$ and $A_{\aleph}^{(2)} \subseteq_{R} A_{\aleph}$, then $A_{\aleph}^{(1)} \subseteq_{R} A_{\aleph}$

(2) If $A_{\aleph} \subseteq_{R} A_{\aleph}^{(1)}$ and $A_{\aleph} \subseteq_{R} A_{\aleph}^{(2)}$, then $A_{\aleph} \subseteq_{R} A_{\aleph}^{(1)} \cap_{R} A_{\aleph}^{(2)}$

(3) If $A_{\aleph}^{(1)} \subseteq_{R} A_{\aleph}$ and $A_{\aleph}^{(2)} \subseteq_{R} A_{\aleph}$, then $A_{\aleph}^{(1)} \cup_{R} A_{\aleph}^{(2)} \subseteq_{R} A_{\aleph}$

(4) If $A_{\aleph}^{(1)} \subseteq_{R} A_{\aleph}$ and $A_{\aleph}^{(2)} \subseteq_{R} B_{\aleph}$, then $A_{\aleph}^{(1)} \cup_{R} A_{\aleph}^{(2)} \subseteq_{R} A_{\aleph}$ $\cup_{R} B_{\aleph}$ and $A_{\aleph}^{(1)} \cap_{R} A_{\aleph}^{(2)} \subseteq_{R} A_{\aleph} \cap_{R} B_{\aleph}$

Theorem 10. Let $A_{\aleph}, A_{\aleph}^{(1)}$, and $A_{\aleph}^{(2)}$ be the usual CmPFS. Then,

(1) $A_{\aleph}^{(1)} \subseteq_{R} A_{\aleph}^{(2)} \Leftrightarrow\left(A_{\aleph}^{(2)}\right)^{c} \subseteq_{R}\left(A_{\aleph}^{(1)}\right)^{c}$

(2) $\left(A_{\aleph}^{(1)} \oplus_{R} A_{\aleph}^{(2)}\right)^{c}=\left(A_{\aleph}^{(1)}\right)^{c} \otimes_{R}\left(A_{\aleph}^{(2)}\right)^{c}$

(3) $\left(A_{\aleph}^{(1)} \otimes_{R} A_{\aleph}^{(2)}\right)^{c}=\left(A_{\aleph}^{(1)}\right)^{c} \oplus_{R}\left(A_{\aleph}^{(2)}\right)^{c}$

Theorem 11. Let $A_{\aleph}, A_{\aleph}^{(1)}$, and $A_{\aleph}^{(2)}$ be the usual CmPFS. Then,

(1) $A_{\aleph}^{(1)} \subseteq_{R} A_{\aleph}^{(2)} \Leftrightarrow \varsigma\left(A_{\aleph}^{(1)}\right) \subseteq_{R} \varsigma\left(A_{\aleph}^{(2)}\right)$

(2) $\varsigma\left(A_{\aleph}^{(1)} \oplus_{R} A_{\aleph}^{(2)}\right)=\left(\left(\left(A_{\aleph}^{(1)} \oplus_{R} A_{\aleph}^{(2)}\right)^{c}\right)^{\varsigma}\right)^{c}$

(3) $\varsigma\left(A_{\aleph}^{(1)} \otimes_{R} A_{\aleph}^{(2)}\right)=\left(\left(\left(A_{\aleph}^{(1)} \otimes_{R} A_{\aleph}^{(2)}\right)^{c}\right)^{\varsigma}\right)^{c}$

(4) $\left(A_{\aleph}^{(1)} \otimes_{R} A_{\aleph}^{(2)}\right)^{\varsigma}=\left(A_{\aleph}^{(1)}\right)^{\varsigma} \otimes_{R}\left(A_{\aleph}^{(2)}\right)^{\varsigma}$

(5) $\varsigma\left(A_{\aleph}^{(1)}\right) \otimes_{R} \varsigma\left(A_{\aleph}^{(2)}\right)=\left(\left(\left(A_{\aleph}^{(1)}\right)^{c}\right)^{\varsigma} \oplus_{R}\left(\left(A_{\aleph}^{(2)}\right)^{c}\right)^{\varsigma}\right)^{c}$
(6) $\varsigma\left(A_{\aleph}^{(1)} \oplus_{R} A_{\aleph}^{(2)}\right)=\varsigma\left(A_{\aleph}^{(1)}\right) \oplus_{R} \varsigma\left(A_{\aleph}^{(2)}\right)$

Proof. Straightforward.

3.1. Cubic m-Polar Fuzzy Relations. Now, we define cubic $m$-polar fuzzy relations (CmPFRs) and give their different types.

Definition 10. Let $\mathscr{X}_{c}$ and $\mathscr{Y}_{c}$ be the two crisp universes. A CmPFR $\mathscr{X}_{c} \longrightarrow \mathscr{Y}_{c}$ is an entity given by

$$
R_{X_{c} \longrightarrow \mathscr{Y}_{c}}=\left\{\left\langle(u, v),\left[\eta_{i}^{-}, \eta_{i}^{+}\right], \eta_{i}\right\rangle_{i=1}^{m}: u \in \mathscr{X}_{c}, v \in \mathscr{Y}_{c}\right\},
$$

where $0 \leq \eta_{i}^{-}, \eta_{i}^{+}, \eta_{i} \leq 1$, and $\eta_{i}^{-} \leq \eta_{i}^{+}$for all $i=1,2, \ldots, m$.

Definition 11. The different types of the CmPFRs are given as follows:

(i) A CmPFR $R_{X_{c} \longrightarrow \mathscr{Y}_{c}}$ is said to be a reflexive if $\left[\eta_{i}^{-}, \eta_{i}^{+}\right]=1$ and $\eta_{i}=0$ for all $i$ and $u \in \mathscr{X}_{c}, v \in \mathscr{Y}_{c}$

(ii) $R_{X_{c}} \longrightarrow \mathscr{Y}_{c}$ is said to be CmPFR of symmetric type if $R_{X_{c} \longrightarrow \mathscr{Y}_{c}}(u, v)=R_{X_{c}} \longrightarrow \mathscr{Y}_{c}(v, u)$ for all $u \in \mathscr{X}_{c}, v \in \mathscr{Y}_{c}$

(iii) $R_{X_{c}} \rightarrow \mathscr{y}_{c}$ is said to be CmPFR of transitive type if $R_{X_{c}} \longrightarrow \mathscr{Y}_{c}(u, w) \geq{ }_{P} \vee_{v}\left(R_{X_{c}} \longrightarrow \mathscr{Y}_{c}(u, v) \wedge\right.$

$\left.R_{X_{c}} \rightarrow \mathscr{Y}_{c}(v, w)\right)$

\section{Correlation Measures for CmPFSs}

In this section, we define correlation measure for two cubic $m$-polar fuzzy sets (CmPFSs) which can describe the informational settings for CmPFSs to vary or tend to vary together. If two settings tend to vary in some direction, simultaneously, they are said to be correlated. If both of them increase/decrease, simultaneously, they are positively correlated, otherwise negatively correlated. Correlation actually measures the closeness of the two variables. We establish certain properties of proposed correlation measures and also discuss novelty and effectiveness of these measures.

Definition 12. Let $A_{\aleph}=\left\{\left\langle\kappa,\left[\eta_{i}^{-}(\kappa), \eta_{i}^{+}(\kappa)\right], \eta_{i}(\kappa)\right\rangle_{i=1}^{m}: \kappa \epsilon\right.$ $\aleph\}$ be a CmPFS over the discourse $\aleph$. The average or mean of $A_{\aleph}$ can be defined as follows:

$$
\begin{aligned}
\overline{A_{\aleph}} & =\left\langle\left[\overline{\eta_{i}^{-}}, \overline{\eta_{i}^{+}}\right], \overline{\eta_{i}}\right\rangle_{i=1}^{m} \\
& =\left\langle\left[\frac{1}{|\aleph|} \sum_{\kappa} \eta_{i}^{-}(\kappa), \frac{1}{|\aleph|} \sum_{\kappa} \eta_{i}^{+}(\kappa)\right], \frac{1}{|\aleph|} \sum_{\kappa} \eta_{i}(\kappa)\right\rangle_{i=1}^{m} .
\end{aligned}
$$

If we take the universe $\aleph=\left\{\kappa_{1}, \kappa_{2}, \kappa_{3}, \kappa_{4}\right\}$ and CmPFS 


$$
A_{\mathrm{N}}=\kappa_{\kappa_{3}}^{\kappa_{1}}\left(\begin{array}{l}
{\left[\eta_{11}^{-}, \eta_{11}^{+}\right],\left[\eta_{12}^{-}, \eta_{12}^{+}\right],\left[\eta_{13}^{-}, \eta_{13}^{+}\right], \eta_{14}, \eta_{15}, \eta_{16}} \\
\left.\kappa_{21}^{-}, \eta_{21}^{+}\right],\left[\eta_{22}^{-}, \eta_{22}^{+}\right],\left[\eta_{23}^{-}, \eta_{23}^{+}\right], \eta_{24}, \eta_{25}, \eta_{26} \\
{\left[\eta_{31}^{-}, \eta_{31}^{+}\right],\left[\eta_{32}^{-}, \eta_{32}^{+}\right],\left[\eta_{33}^{-}, \eta_{33}^{+}\right], \eta_{34}, \eta_{35}, \eta_{36}} \\
{\left[\eta_{41}^{-}, \eta_{41}^{+}\right],\left[\eta_{42}^{-}, \eta_{42}^{+}\right],\left[\eta_{43}^{-}, \eta_{43}^{+}\right], \eta_{44}, \eta_{45}, \eta_{46}}
\end{array}\right),
$$

then the average of $A_{\aleph}$ is given by $\overline{A_{\aleph}}=\left[\overline{\eta_{1}^{-}}, \overline{\eta_{1}^{+}}\right]$, $\left[\overline{\eta_{2}^{-}}, \overline{\eta_{2}^{+}}\right],\left[\overline{\eta_{3}^{-}}, \overline{\eta_{3}^{+}}\right], \overline{\eta_{4}}, \overline{\eta_{5}}, \overline{\eta_{6}}$, where

$$
\begin{aligned}
& \overline{\overline{\eta_{1}}}=\frac{1}{4} \sum_{j=1}^{4} \eta_{j 1}^{-}, \\
& \overline{\eta_{1}^{+}}=\frac{1}{4} \sum_{j=1}^{4} \eta_{j 1}^{+}, \\
& \overline{\overline{\eta_{2}}}=\frac{1}{4} \sum_{j=1}^{4} \eta_{j 2}^{-}, \\
& \overline{\eta_{2}^{+}}=\frac{1}{4} \sum_{j=1}^{4} \eta_{j 2}^{+}, \\
& \overline{\eta_{3}^{-}}=\frac{1}{4} \sum_{j=1}^{4} \eta_{j 3}^{-}, \\
& \overline{\eta_{3}^{+}}=\frac{1}{4} \sum_{j=1}^{4} \eta_{j 3}^{+}, \\
& \overline{\eta_{4}}=\frac{1}{4} \sum_{j=1}^{4} \eta_{j 4}, \\
& \overline{\eta_{5}}=\frac{1}{4} \sum_{j=1}^{4} \eta_{j 5}, \\
& \overline{\eta_{6}}=\frac{1}{4} \sum_{j=1}^{4} \eta_{j 6} .
\end{aligned}
$$

\section{Definition 13. Let}

$$
\begin{aligned}
& A_{\mathrm{N}}=\underset{\kappa_{2}}{\kappa_{2}} \begin{array}{c}
\kappa_{1} \\
\vdots
\end{array}\left(\begin{array}{c}
{\left[\eta_{11}^{-}, \eta_{11}^{+}\right],\left[\eta_{12}^{-}, \eta_{12}^{+}\right], \ldots,\left[\eta_{1 m}^{-}, \eta_{1 m}^{+}\right], \eta_{11}, \eta_{12}, \ldots, \eta_{1 m}} \\
{\left[\eta_{21}^{-}, \eta_{21}^{+}\right],\left[\eta_{22}^{-}, \eta_{22}^{+}\right], \ldots,\left[\eta_{2 m}^{-}, \eta_{2 m}^{+}\right], \eta_{21}, \eta_{22}, \ldots, \eta_{2 m}} \\
\ldots \\
{\left[\eta_{n 1}^{-}, \eta_{n 1}^{+}\right],\left[\eta_{n 2}^{-}, \eta_{n 2}^{+}\right], \ldots,\left[\eta_{n m}^{-}, \eta_{n m}^{+}\right], \eta_{n 1}, \eta_{n 2}, \ldots, \eta_{n m}}
\end{array}\right), \\
& B_{\aleph}=\begin{array}{c}
\kappa_{2} \\
\vdots \\
\kappa_{n}
\end{array}\left(\begin{array}{c}
{\left[\zeta_{11}^{-}, \zeta_{11}^{+}\right],\left[\zeta_{12}^{-}, \zeta_{12}^{+}\right], \ldots,\left[\zeta_{1 m}^{-}, \zeta_{1 m}^{+}\right], \zeta_{11}, \zeta_{12}, \ldots, \zeta_{1 m}} \\
{\left[\zeta_{21}^{+}\right],\left[\zeta_{22}^{-}, \zeta_{22}^{+}\right], \ldots,\left[\zeta_{2 m}^{-}, \zeta_{2 m}^{+}\right], \zeta_{21}, \zeta_{22}, \ldots, \zeta_{2 m}} \\
\ldots
\end{array}\right)
\end{aligned}
$$

be the two CmPFSs over $\aleph=\left\{\kappa_{1}, \kappa_{2}, \kappa_{3}, \ldots, \kappa_{n}\right\}$. The left and right relative informational coefficients (RIC) of $A_{\aleph}$ over $B_{\aleph}$ are, respectively, defined by

$$
\begin{aligned}
I_{j\left(A_{\aleph} \longrightarrow B_{\aleph}\right.}^{-} & =\sum_{i=1}^{n}\left(\eta_{i j}^{-}-\overline{\eta_{j}^{-}}\right)\left(\zeta_{i j}^{-}-\overline{\zeta_{j}^{-}}\right), \\
I_{j\left(A_{\aleph} \longrightarrow B_{\aleph}\right)}^{+} & =\sum_{i=1}^{n}\left(\eta_{i j}^{+}-\overline{\eta_{j}^{+}}\right)\left(\zeta_{i j}^{+}-\overline{\zeta_{j}^{+}}\right), \quad j=1,2, \ldots, m,
\end{aligned}
$$

and relative noninformational coefficients (RNICs) by

$$
I_{j\left(A_{\aleph} \longrightarrow B_{\aleph}\right)}=\sum_{i=1}^{n}\left(\eta_{i j}-\overline{\eta_{j}}\right)\left(\zeta_{i j}-\overline{\zeta_{j}}\right), \quad j=1,2, \ldots, m .
$$

Similarly, the left and right informational coefficients of $A_{\aleph}$ are, respectively, given by

$$
\begin{aligned}
& \mathbb{C}_{j}^{-}\left(A_{\aleph}\right)=\sum_{i=1}^{n}\left(\eta_{i j}^{-}-\overline{\eta_{j}^{-}}\right)^{2}, \\
& \mathbb{C}_{j}^{+}\left(A_{\aleph}\right)=\sum_{i=1}^{n}\left(\eta_{i j}^{+}-\overline{\eta_{j}^{+}}\right)^{2},
\end{aligned}
$$

and noninformational coefficient by

$$
\mathbb{C}_{j}\left(A_{\aleph}\right)=\sum_{i=1}^{n}\left(\eta_{i j}-\overline{\eta_{j}}\right)^{2}, \quad j=1,2, \ldots, m .
$$

Proposition 3. The RICs/RNICs and the ICs/NICs for the CmPFSs $A_{\aleph}$ and $B_{\aleph}$ follow the following properties:

(a) $I_{j\left(A_{\aleph} \longrightarrow A_{\aleph}\right)}^{-}=\mathbb{C}_{j}^{-}\left(A_{\aleph}\right), \quad I_{j\left(A_{\aleph} \longrightarrow A_{\aleph}\right)}^{+}=\mathbb{C}_{j}^{+}\left(A_{\aleph}\right), \quad I_{j}$ $\left(A_{\aleph} \longrightarrow A_{\aleph}\right)=\mathbb{C}_{j}\left(A_{\aleph}\right)$

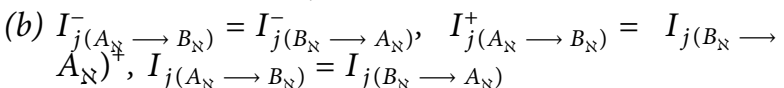

(c) $\begin{aligned}\left|I_{j\left(A_{\aleph} \longrightarrow B_{\aleph}\right.}^{-}\right| \leq \sqrt{\mathbb{C}_{j}^{-}\left(A_{\aleph}\right) \mathbb{C}_{j}^{-}\left(B_{\aleph}\right)}, \quad\left|I_{j\left(A_{\aleph} \longrightarrow B_{\aleph}\right)}^{+}\right| \leq \\ \sqrt{\mathbb{C}_{j}^{+}\left(A_{\aleph}\right) \mathbb{C}_{j}^{+}\left(B_{\aleph}\right)},\left|I_{j\left(A_{\aleph} \longrightarrow B_{\aleph}\right)}\right| \leq \sqrt{\mathbb{C}_{j}\left(A_{\aleph}\right) \mathbb{C}_{j}\left(B_{\aleph}\right)}\end{aligned}$

Proof. (a) and (b) are easy to address.

(c) The well-known Cauchy-Schwarz inequality states that $\left(\sum_{q=1}^{k} u_{q} v_{q}\right)^{2} \leq\left(\sum_{q=1}^{k} u_{q}^{2}\right)\left(\sum_{q=1}^{k} v_{q}^{2}\right)$ for $u_{q}, v_{q} \in R$. We will use the above inequality to prove our result.

Consider

$$
\begin{aligned}
\left(I_{j\left(A_{\aleph} \rightarrow B_{\aleph}\right)}\right)^{2} & =\left(\sum_{i=1}^{n}\left(\eta_{i j}^{-}-\overline{\eta_{j}^{-}}\right)\left(\zeta_{i j}^{-}-\overline{\zeta_{j}^{-}}\right)\right)^{2} \\
& \leq \sum_{i=1}^{n}\left(\eta_{i j}^{-}-\overline{\eta_{j}^{-}}\right)^{2} \sum_{i=1}^{n}\left(\bar{\zeta}_{i j}^{-}-\overline{\zeta_{j}^{-}}\right)^{2} \\
& =\mathbb{C}_{j}^{-}\left(A_{\aleph}\right) \mathbb{C}_{j}^{-}\left(B_{\aleph}\right) \\
& \Rightarrow\left|I_{j}^{-}\left(A_{\aleph} \rightarrow B_{\aleph}\right)\right| \leq \sqrt{\mathbb{C}_{j}^{-}\left(A_{\aleph}\right) \mathbb{C}_{j}^{-}\left(B_{\aleph}\right)}, \quad \text { for all } j .
\end{aligned}
$$

The other two results are similar. 
Definition 14. If $A_{\aleph}$ and $B_{\aleph}$ are the two CmPFSs with the usual notations, then their relative correlation coefficient (RCC) is given by

$$
\partial\left(A_{\aleph}, B_{\aleph}\right)=\frac{1}{3 m}\left(\sum_{j=1}^{m} \partial_{j}^{-}\left(A_{\aleph}, B_{\aleph}\right)+\sum_{j=1}^{m} \partial_{j}^{+}\left(A_{\aleph}, B_{\aleph}\right)+\sum_{j=1}^{m} \partial_{j}\left(A_{\aleph}, B_{\aleph}\right)\right)
$$

where

$$
\begin{aligned}
\partial_{j}^{-}\left(A_{\aleph}, B_{\aleph}\right) & =\frac{\sum_{i=1}^{n}\left(\eta_{i j}^{-}-\overline{\eta_{j}^{-}}\right)\left(\zeta_{i j}^{-}-\overline{\zeta_{j}^{-}}\right)}{\sqrt{\sum_{i=1}^{n}\left(\eta_{i j}^{-}-\overline{\left(\eta_{j}^{-}\right)}\right)^{2} \sum_{i=1}^{n}\left(\zeta_{i j}^{-}-\overline{\left(\zeta_{j}^{-}\right)}\right)^{2}}}, \\
\partial_{j}^{+}\left(A_{\aleph}, B_{\aleph}\right) & =\frac{\sum_{i=1}^{n}\left(\eta_{i j}^{+}-\overline{\eta_{j}^{+}}\right)\left(\zeta_{i j}^{+}-\overline{\zeta_{j}^{+}}\right)}{\sqrt{\sum_{i=1}^{n}\left(\eta_{i j}^{+}-\overline{\left(\eta_{j}^{+}\right)}\right)^{2} \sum_{i=1}^{n}\left(\zeta_{i j}^{+}-\overline{\left(\zeta_{j}^{+}\right)}\right)^{2}}}, \\
\partial_{j}\left(A_{\aleph}, B_{\aleph}\right) & =\frac{\sum_{i=1}^{n}\left(\eta_{i j}-\overline{\eta_{j}}\right)\left(\zeta_{i j}-\overline{\zeta_{j}}\right)}{\sqrt{\sum_{i=1}^{n}\left(\eta_{i j}-\overline{\left(\eta_{j}\right)}\right)^{2} \sum_{i=1}^{n}\left(\zeta_{i j}-\overline{\left(\zeta_{j}\right)}\right)^{2}}}
\end{aligned}
$$

Theorem 12. For the CmPFSs $A_{\aleph}$ and $B_{\aleph}$, we have

(a) The correlation $\partial$ is symmetrical, that is, $\partial\left(A_{\aleph}, B_{\aleph}\right)=\partial\left(B_{\aleph}, A_{\aleph}\right)$

(b) The correlation $\partial$ finds the interval $[-1,1]$, that is, $-1 \leq \partial\left(A_{\aleph}, B_{\aleph}\right) \leq 1$

(c) $\partial\left(A_{\aleph}, B_{\aleph}\right)=1$ if $A_{\aleph}=B_{\aleph}$

(d) $\partial\left(A_{\aleph}, B_{\aleph}\right)=1$ if $A_{\aleph}=(\lambda) B_{\aleph}, \lambda>0$

(here, $(\lambda) B_{\aleph}$ means $\left\{\left\langle\left[\lambda \zeta_{i j}^{-}, \lambda \zeta_{i j}^{+}\right], \lambda \zeta_{i j}\right\rangle_{j=1}^{m}\right)$

(e) $\partial\left(A_{\aleph}, B_{\aleph}\right)=-1$ if $A_{\aleph}=\lambda\left(B_{\aleph}\right)^{c}, \lambda>0$

Proof

(a) Straightforward.

(b) From Proposition 3, we have

$j=1,2, \ldots, m$.

$$
\begin{aligned}
&\left|I_{j\left(A_{\aleph} \longrightarrow B_{\aleph}\right)}^{-}\right| \leq \sqrt{\mathbb{C}_{j}^{-}\left(A_{\aleph}\right) \mathbb{C}_{j}^{-}\left(B_{\aleph}\right)} \\
& \Rightarrow-\sqrt{\mathbb{C}_{j}^{-}\left(A_{\aleph}\right) \mathbb{C}_{j}^{-}\left(B_{\aleph}\right)} \leq I_{j}^{-}\left(A_{\aleph} \longrightarrow B_{\aleph}\right) \leq \sqrt{\mathbb{C}_{j}^{-}\left(A_{\aleph}\right) \mathbb{C}_{j}^{-}\left(B_{\aleph}\right)} \\
& \Rightarrow-1 \leq \frac{I_{j}^{-}\left(A_{\aleph} \longrightarrow B_{\aleph}\right)}{\sqrt{\mathbb{C}_{j}^{-}\left(A_{\aleph}\right) \mathbb{C}_{j}^{-}\left(B_{\aleph}\right)}} \leq 1 .
\end{aligned}
$$

Hence, $-1 \leq \partial_{j}^{-}\left(A_{\aleph}, B_{\aleph}\right) \leq 1$.

Similarly, $-1 \leq \partial_{j}^{+}\left(A_{\aleph}, B_{\aleph}\right) \leq 1$ and $-1 \leq \partial_{j}\left(A_{\aleph}, B_{\aleph}\right)$ $\leq 1$, for all $j=1,2, \ldots, m$.

Consequently, $-1 \leq \partial\left(A_{\aleph}, B_{\aleph}\right) \leq 1$, being the mean of the quantities between -1 and 1 .

(c) If $A_{\aleph}=B_{\aleph}$, then $\eta_{i j}^{-}=\zeta_{i j}^{-}, \eta_{i j}^{+}=\zeta_{i j}^{+}, \eta_{i j}=\zeta_{i j}$.

Therefore, $\partial_{j}^{-}\left(A_{\aleph}, B_{\aleph}\right)=\left(\sum_{i=1}^{n}\left(\eta_{i j}^{-}-\overline{\eta_{j}^{-}}\right)\left(\zeta_{i j}^{-}-\overline{\zeta_{j}^{-}}\right) /\right.$ $\left.\sqrt{\sum_{i=1}^{n}\left(\eta_{i j}^{-}-\overline{\left(\eta_{j}^{-}\right)}\right)^{2}} \quad \sum_{i=1}^{n}\left(\zeta_{i j}^{-}-\overline{\left(\zeta_{j}^{-}\right)}\right)^{2}\right)=\left(\sum_{i=}\right.$ $1^{n}\left(\eta_{i j}^{-}-\overline{\eta_{j}^{-}}\right)\left(\eta_{i j}^{-}-\overline{\eta_{j}^{-}}\right) /$

$\sqrt{\left.\sum_{i=1}^{n}\left(\eta_{i j}^{-}-\overline{\left(\eta_{j}^{-}\right)}\right)^{2} \sum_{i=1}^{n}\left(\eta_{i j}^{-}-\overline{\left(\eta_{j}^{-}\right)}\right)^{2}\right)=1}$.

Similarly, $\partial_{j}^{+}\left(A_{\aleph}, B_{\aleph}\right)=1$ and $\partial_{j}\left(A_{\aleph}, B_{\aleph}\right)=1$, and hence, $\partial\left(A_{\aleph}, B_{\aleph}\right)=1$.

(d) If $A_{\aleph}=(\lambda) B_{\aleph}, \lambda>0$, then $\eta_{i j}^{-}=\lambda \zeta_{i j}^{-}, \eta_{i j}^{+}=\lambda \zeta_{i j}^{+}$, and $\eta_{i j}=\lambda \zeta_{i j}$. Therefore,

$$
\partial_{j}^{-}\left(A_{\aleph}, B_{\aleph}\right)=\frac{\sum_{i=1}^{n}\left(\eta_{i j}^{-}-\overline{\eta_{j}^{-}}\right)\left(\zeta_{i j}^{-}-\overline{\zeta_{j}^{-}}\right)}{\sqrt{\sum_{i=1}^{n}\left(\eta_{i j}^{-}-\overline{\left(\eta_{j}^{-}\right)}\right)^{2} \sum_{i=1}^{n}\left(\zeta_{i j}^{-}-\overline{\left(\zeta_{j}^{-}\right)}\right)^{2}}}=\frac{\sum_{i=1}^{n}\left(\lambda \zeta_{i j}^{-}-\overline{\lambda \zeta_{i j}^{-}}\right)\left(\zeta_{i j}^{-}-\overline{\zeta_{j}^{-}}\right)}{\sqrt{\sum_{i=1}^{n}\left(\lambda \zeta_{i j}^{-}-\overline{\left(\lambda \zeta_{i j}^{-}\right.}\right)^{2} \sum_{i=1}^{n}\left(\zeta_{i j}^{-}-\overline{\left(\zeta_{j}^{-}\right)}\right)^{2}}}=1
$$

Similarly, $\partial_{j}^{+}\left(A_{\aleph}, B_{\aleph}\right)=1, \quad \partial_{j}\left(A_{\aleph}, B_{\aleph}\right)=1, \quad$ and hence, $\partial\left(A_{\aleph}, B_{\aleph}\right)=1$. (e) If $A_{\aleph}=\lambda\left(B_{\aleph}\right)^{c}, \quad \lambda>0, \quad$ then $\eta_{i j}^{-}=\lambda\left(1-\zeta_{i j}^{-}\right)$, $\eta_{i j}^{+}=\lambda\left(1-\zeta_{i j}^{+}\right)$, and $\eta_{i j}=\lambda\left(1-\zeta_{i j}\right)$. 
Therefore, $\overline{\eta_{j}^{-}}=1-\overline{\zeta_{j}^{-}}$and

$$
\begin{aligned}
\partial_{j}^{-}\left(A_{\aleph}, B_{\aleph}\right) & =\frac{\sum_{i=1}^{n}\left(\eta_{i j}^{-}-\overline{\eta_{j}}\right)\left(\zeta_{i j}^{-}-\overline{\zeta_{j}^{-}}\right)}{\sqrt{\sum_{i=1}^{n}\left(\overline{\eta_{i j}^{-}}-\overline{\left(\eta_{j}^{-}\right)}\right)^{2} \sum_{i=1}^{n}\left(\zeta_{i j}^{-}-\overline{\left(\zeta_{j}^{-}\right)}\right)^{2}}}=\frac{\sum_{i=1}^{n}\left(\lambda\left(1-\zeta_{i j}^{-}\right)-\lambda\left(1-\overline{\zeta_{i j}^{-}}\right)\right)\left(\zeta_{i j}^{-}-\overline{\zeta_{j}^{-}}\right)}{\sqrt{\left.\sum_{i=1}^{n}\left(\lambda\left(1-\zeta_{i j}^{-}\right)-\lambda\left(1-\overline{\zeta_{i j}^{-}}\right)\right)^{2} \sum_{i=1}^{n}\left(\zeta_{i j}^{-}-\overline{\left(\zeta_{j}^{-}\right.}\right)\right)^{2}}} \\
& =-\frac{\sum_{i=1}^{n}\left(\zeta_{i j}^{-}-\overline{\zeta_{i j}^{-}}\right)\left(\zeta_{i j}^{-}-\overline{\zeta_{j}^{-}}\right)}{\sqrt{\sum_{i=1}^{n}\left(\zeta_{i j}^{-}-\overline{\left(\zeta_{j}^{-}\right)}\right)^{2} \sum_{i=1}^{n}\left(\zeta_{i j}^{-}-\overline{\left(\zeta_{j}^{-}\right)}\right)^{2}}}=-\frac{\sum_{i=1}^{n}\left(\zeta_{i j}^{-}-\overline{\zeta_{i j}^{-}}\right)^{2}}{\sqrt{\sum_{i=1}^{n}\left(\zeta_{i j}^{-}-\overline{\left(\zeta_{j}^{-}\right)}\right)^{2} \sum_{i=1}^{n}\left(\zeta_{i j}^{-}-\overline{\left.\left(\zeta_{j}^{-}\right)\right)^{2}}\right.}}=-1 .
\end{aligned}
$$

Similarly, $\partial_{j}^{+}\left(A_{\aleph}, B_{\aleph}\right)=-1, \quad \partial_{j}\left(A_{\aleph}, B_{\aleph}\right)=-1, \quad$ and Definition 15. For the CmPFSs $A_{\aleph}$ and $B_{\aleph}$, the RCC may hence, $\partial\left(A_{\aleph}, B_{\aleph}\right)=-1$.

$$
\sigma\left(A_{\aleph}, B_{\aleph}\right)=\frac{1}{3 m}\left(\sum_{j=1}^{m} \sigma_{j}^{-}\left(A_{\aleph}, B_{\aleph}\right)+\sum_{j=1}^{m} \sigma_{j}^{+}\left(A_{\aleph}, B_{\aleph}\right)+\sum_{j=1}^{m} \sigma_{j}\left(A_{\aleph}, B_{\aleph}\right)\right),
$$

where

$$
\begin{aligned}
& \sigma_{j}^{-}\left(A_{\aleph}, B_{\aleph}\right)=\frac{\sum_{i=1}^{n}\left(\overline{\eta_{i j}^{-}}-\overline{\eta_{j}^{-}}\right)\left(\zeta_{i j}^{-}-\overline{\zeta_{j}^{-}}\right)}{\max \left\{\sum_{i=1}^{n}\left(\overline{\eta_{i j}^{-}}-\overline{\left(\eta_{j}^{-}\right)}\right)^{2}, \sum_{i=1}^{n}\left(\overline{\zeta_{i j}^{-}}-\overline{\left(\zeta_{j}^{-}\right)}\right)^{2}\right\}}, \\
& \sigma_{j}^{+}\left(A_{\aleph}, B_{\aleph}\right)=\frac{\sum_{i=1}^{n}\left(\eta_{i j}^{+}-\overline{\eta_{j}^{+}}\right)\left(\zeta_{i j}^{+}-\overline{\zeta_{j}^{+}}\right)}{\max \left\{\sum_{i=1}^{n}\left(\eta_{i j}^{+}-\overline{\left(\eta_{j}^{+}\right)}\right)^{2}, \sum_{i=1}^{n}\left(\zeta_{i j}^{+}-\overline{\left(\zeta_{j}^{+}\right)}\right)^{2}\right\}}, \\
& \sigma_{j}\left(A_{\aleph}, B_{\aleph}\right)=\frac{\sum_{i=1}^{n}\left(\eta_{i j}-\overline{\eta_{j}}\right)\left(\zeta_{i j}-\overline{\zeta_{j}}\right)}{\max \left\{\sum_{i=1}^{n}\left(\eta_{i j}-\overline{\left(\eta_{j}\right)}\right)^{2}, \sum_{i=1}^{n}\left(\zeta_{i j}-\overline{\left(\zeta_{j}\right)}\right)^{2}\right\}},
\end{aligned}
$$

Theorem 13. For the CmPFSs $A_{\aleph}$ and $B_{\aleph}$, we have

(a) The correlation $\sigma$ is symmetrical, that is, $\sigma\left(A_{\aleph}\right.$, $\left.B_{\aleph}\right)=\sigma\left(B_{\aleph}, A_{\aleph}\right)$

(b) The correlation $\sigma$ finds the interval $[-1,1]$, that is, $-1 \leq \sigma\left(A_{\aleph}, B_{\aleph}\right) \leq 1$

(c) $\sigma\left(A_{\aleph}, B_{\aleph}\right)=1$ if $A_{\aleph}=B_{\aleph}$

Proof

(a) and (b) are straightforward.

(c) Suppose $A_{\aleph}=B_{\aleph}$. Then, $\eta_{i j}^{-}=\zeta_{i j}^{-}, \eta_{i j}^{+}=\zeta_{i j}^{+}$, and $\eta_{i j}=\zeta_{i j}$. In this case,

$j=1,2, \ldots, m$.

$$
\begin{aligned}
\sigma_{j}^{-}\left(A_{\aleph}, B_{\aleph}\right) & =\frac{\sum_{i=1}^{n}\left(\overline{\eta_{i j}^{-}}-\overline{\eta_{j}^{-}}\right)\left(\zeta_{i j}^{-}-\overline{\zeta_{j}^{-}}\right)}{\max \left\{\sum_{i=1}^{n}\left(\eta_{i j}^{-}-\overline{\left(\eta_{j}^{-}\right)}\right)^{2}, \sum_{i=1}^{n}\left(\zeta_{i j}^{-}-\overline{\left(\zeta_{j}^{-}\right)}\right)^{2}\right\}}=\frac{\sum_{i=1}^{n}\left(\zeta_{i j}^{-}-\overline{\zeta_{j}^{-}}\right)\left(\zeta_{i j}^{-}-\overline{\zeta_{j}^{-}}\right)}{\max \left\{\sum_{i=1}^{n}\left(\zeta_{i j}^{-}-\overline{\left(\zeta_{j}^{-}\right)}\right)^{2}, \sum_{i=1}^{n}\left(\zeta_{i j}^{-}-\overline{\left(\zeta_{j}^{-}\right.}\right)^{2}\right\}} \\
& =\frac{\sum_{i=1}^{n}\left(\zeta_{i j}^{-}-\overline{\zeta_{j}^{-}}\right)^{2}}{\max \left\{\sum_{i=1}^{n}\left(\zeta_{i j}^{-}-\overline{\left(\zeta_{j}^{-}\right)}\right)^{2}, \sum_{i=1}^{n}\left(\zeta_{i j}^{-}-\overline{\left(\zeta_{j}^{-}\right)}\right)^{2}\right\}}=1 .
\end{aligned}
$$

Similarly, $\sigma_{j}^{+}\left(A_{\aleph}, B_{\aleph}\right)=1$ and $\sigma_{j}\left(A_{\aleph}, B_{\aleph}\right)=1$. Hence, $\sigma\left(A_{\aleph}, B_{\aleph}\right)=1$.

In most real-world problems, two major aspects should be addressed, that is, uncertainty and weights. While dealing with such problems, uncertainty is a key factor which can affect our decisions and analysis. Besides, most of the uncertain mathematical models are effected by weights. To deal with such problems, we define weighted CCs.

Definition 16. Let $A_{\aleph}$ and $B_{\aleph}$ be the two CmPFSs (usual notation followed), and the alternatives $\kappa_{1}, \kappa_{2}, \ldots, \kappa_{n}$ are affected by the weights $\omega_{1}, \omega_{2}, \ldots, \omega_{n}$ with the condition 
$\sum_{i=1}^{n} \omega_{i}=1$ and $0 \leq \omega_{i} \leq 1$. We define relative informational weighted coefficients (RIWCs) of $A_{\aleph}$ over $B_{\aleph}$ by

$$
\begin{aligned}
\left.(\text { Left RIWC })^{\omega} I_{j\left(A_{\aleph}\right.}^{-} \longrightarrow B_{\aleph}\right) & =\sum_{i=1}^{n} \omega_{i}\left(\eta_{i j}^{-}-\overline{\eta_{j}}\right)\left(\zeta_{i j}^{-}-\overline{\zeta_{j}^{-}}\right), \\
\left(\operatorname { R i g h t ~ R I W C } { } ^ { \omega } I _ { j } ^ { + } \left(A_{\aleph} \longrightarrow B_{\aleph}\right.\right. & =\sum_{i=1}^{n} \omega_{i}\left(\eta_{i j}^{+}-\overline{\eta_{j}^{+}}\right)\left(\zeta_{i j}^{+}-\overline{\zeta_{j}^{+}}\right), \quad j=1,2, \ldots, m,
\end{aligned}
$$

and the relative noninformational weighted coefficients (RNIWCs) by

$$
{ }^{\omega} I_{j\left(A_{\aleph} \longrightarrow B_{\aleph}\right)}=\sum_{i=1}^{n} \omega_{i}\left(\eta_{i j}-\overline{\eta_{j}}\right)\left(\zeta_{i j}-\overline{\zeta_{j}}\right), \quad j=1,2, \ldots, m .
$$

Similarly, the informational (left and right) and noninformational weighted coefficients of $A_{\aleph}$ are, respectively, given by

$$
\begin{aligned}
& { }^{\omega} \mathbb{C}_{j}^{-}\left(A_{\aleph}\right)=\sum_{i=1}^{n} \omega_{i}\left(\eta_{i j}^{-}-\overline{\eta_{j}^{-}}\right)^{2}, \\
& { }^{\omega} \mathbb{C}_{j}^{+}\left(A_{\aleph}\right)=\sum_{i=1}^{n} \omega_{i}\left(\eta_{i j}^{+}-\overline{\eta_{j}^{+}}\right)^{2}, \\
& { }^{\omega} \mathbb{C}_{j}\left(A_{\aleph}\right)=\sum_{i=1}^{n} \omega_{i}\left(\eta_{i j}-\overline{\eta_{j}}\right)^{2}, \quad j=1,2, \ldots, m .
\end{aligned}
$$

Proposition 4. The RIWCs/RNIWCs and the IWCs/NIWCs for the CmPFSs $A_{\aleph}$ and $B_{\aleph}$ follow the following properties:

$\begin{aligned}\left.\text { (a) }{ }^{\omega} I_{j\left(A_{\aleph}\right.}^{-} \longrightarrow A_{\aleph}\right) & \left.={ }^{\omega} \mathbb{C}_{j}^{-}\left(A_{\aleph}\right), \quad{ }^{\omega} I_{j\left(A_{\aleph}\right.}^{+} \longrightarrow A_{\aleph}\right)={ }^{\omega} \mathbb{C}_{j}^{+} \\ & \left.\left(A_{\aleph}\right),{ }^{\omega} I_{j\left(A_{\aleph}\right.} \longrightarrow A_{\aleph}\right)\end{aligned}$

(b) $\left.\left.{ }^{\omega} I_{j\left(A_{\aleph}\right.} \longrightarrow B_{\left.B_{\aleph}\right)}={ }^{\omega} I_{j\left(B_{\aleph}\right.}^{-} \longrightarrow Z_{\aleph}\right){ }^{,}{ }^{\omega} I_{j\left(A_{\aleph}\right.}^{+} \longrightarrow B_{\aleph}\right)={ }^{\omega} I_{j\left(B_{\aleph}\right.}$ $\left.\left.\longrightarrow Z_{\aleph}\right)^{+},{ }^{\omega} I_{j\left(A_{\aleph}\right.} \longrightarrow B_{\aleph}\right)={ }^{\omega} I_{j\left(B_{\aleph} \longrightarrow A_{N}\right)}$

(c) $\left|{ }^{\omega} I_{j\left(A_{\aleph} \longrightarrow B_{\aleph}\right)}\right| \leq \sqrt{{ }^{\omega} \mathbb{C}_{j}^{-}\left(A_{\aleph}\right){ }^{\omega} \mathbb{C}_{j}^{-}\left(B_{\aleph}\right)}, \quad \mid{ }^{\omega} I_{j\left(A_{\aleph} \longrightarrow\right.}$ $\left.B_{\aleph}\right)^{+}\left|\leq \sqrt{\left.{ }^{\omega} \mathbb{C}_{j}^{+}\left(A_{\aleph}\right)\right)^{\omega} \mathbb{C}_{j}^{+}\left(B_{\aleph}\right)}, \quad\right|{ }^{\omega} I_{j\left(A_{\aleph} \longrightarrow B_{\aleph}\right)} \mid \leq$ $\sqrt{{ }^{\omega} \mathbb{C}_{j}\left(A_{\aleph}\right)^{\omega} \mathbb{C}_{j}\left(B_{\aleph}\right)}$
Proof

(a) and (b) are easy to address.

(c) We can prove the results in mirror of Cauchy-Schwarz inequality $\left(\sum_{q=1}^{k} u_{q} v_{q}\right)^{2} \leq\left(\sum_{q=1}^{k} u_{q}^{2}\right)$ $\left(\sum_{q=1}^{k} v_{q}^{2}\right)$ for $u_{q}, v_{q} \in R$.

\section{Consider}

$$
\begin{aligned}
& \left.\left({ }^{\omega} I_{j\left(A_{\aleph}\right.}^{-} \longrightarrow B_{\aleph}\right)\right)^{2}=\left(\sum_{i=1}^{n} \omega_{i}\left(\eta_{i j}^{-}-\overline{\eta_{j}^{-}}\right)\left(\zeta_{i j}^{-}-\overline{\zeta_{j}^{-}}\right)\right)^{2} \\
& =\left(\sum_{i=1}^{n} \sqrt{\omega_{i}}\left(\eta_{i j}^{-}-\overline{\eta_{j}}\right) \sqrt{\omega_{i}}\left(\zeta_{i j}^{-}-\overline{\zeta_{j}^{-}}\right)\right)^{2} \\
& \leq \sum_{i=1}^{n} \omega_{i}\left(\eta_{i j}^{-}-\overline{\eta_{j}^{-}}\right)^{2} \sum_{i=1}^{n} \omega_{i}\left(\zeta_{i j}^{-}-\overline{\zeta_{j}^{-}}\right)^{2} \\
& ={ }^{\omega} \mathbb{C}_{j}^{-}\left(A_{\aleph}\right){ }^{\omega} \mathbb{C}_{j}^{-}\left(B_{\aleph}\right) \\
& \Rightarrow\left|{ }^{\omega} I_{j}^{-}\left(A_{\aleph} \longrightarrow B_{\aleph}\right)\right| \leq \sqrt{{ }^{\omega} \mathbb{C}_{j}^{-}\left(A_{\aleph}\right){ }^{\omega} \mathbb{C}_{j}^{-}\left(B_{\aleph}\right)}, \quad \text { for all } j \text {. }
\end{aligned}
$$

The other two results are similar.

Definition 17. If $A_{\aleph}$ and $B_{\aleph}$ are the two CmPFSs, then their weighted correlation coefficient (WCC) is given by

$$
\partial_{\omega}\left(A_{\aleph}, B_{\aleph}\right)=\frac{1}{3 m}\left(\sum_{j=1}^{m} \omega \partial_{j}^{-}\left(A_{\aleph}, B_{\aleph}\right)+\sum_{j=1}^{m} \omega \partial_{j}^{+}\left(A_{\aleph}, B_{\aleph}\right)+\sum_{j=1}^{m} \omega \partial_{j}\left(A_{\aleph}, B_{\aleph}\right)\right),
$$


where

$$
\begin{aligned}
& { }^{\omega} \partial_{j}^{-}\left(A_{\aleph}, B_{\aleph}\right)=\frac{\sum_{i=1}^{n} \omega_{i}\left(\overline{\eta_{i j}^{-}}-\overline{\overline{\eta_{j}}}\right)\left(\zeta_{i j}^{-}-\overline{\overline{\zeta_{j}}}\right)}{\sqrt{\sum_{i=1}^{n} \omega_{i}\left(\overline{\eta_{i j}^{-}}-\overline{\left(\eta_{j}^{-}\right)}\right)^{2} \sum_{i=1}^{n} \omega_{i}\left(\overline{\left.\left.\zeta_{i j}^{-}-\overline{\left(\zeta_{j}^{-}\right.}\right)\right)^{2}}\right.},} \\
& { }^{\omega} \partial_{j}^{+}\left(A_{\aleph}, B_{\aleph}\right)=\frac{\sum_{i=1}^{n} \omega_{i}\left(\eta_{i j}^{+}-\overline{\eta_{j}^{+}}\right)\left(\zeta_{i j}^{+}-\overline{\zeta_{j}^{+}}\right)}{\sqrt{\sum_{i=1}^{n} \omega_{i}\left(\eta_{i j}^{+}-\overline{\left(\eta_{j}^{+}\right)}\right)^{2} \sum_{i=1}^{n} \omega_{i}\left(\zeta_{i j}^{+}-\overline{\left(\zeta_{j}^{+}\right)}\right)^{2}}}, \\
& { }^{\omega} \partial_{j}\left(A_{\aleph}, B_{\aleph}\right)=\frac{\sum_{i=1}^{n} \omega_{i}\left(\eta_{i j}-\overline{\eta_{j}}\right)\left(\zeta_{i j}-\overline{\zeta_{j}}\right)}{\sqrt{\sum_{i=1}^{n} \omega_{i}\left(\eta_{i j}-\overline{\left(\eta_{j}\right)}\right)^{2} \sum_{i=1}^{n} \omega_{i}\left(\zeta_{i j}-\overline{\left(\zeta_{j}\right)}\right)^{2}}},
\end{aligned}
$$

$j=1,2, \ldots, m$.
Theorem 14. If $A_{\mathrm{N}}$ and $B_{\mathrm{N}}$ are CmPFSs with the preassigned weights $\left(\omega_{1}, \omega_{2}, \ldots, \omega_{n}\right)$, then

(a) the correlation $\partial_{\omega}$ is symmetrical, that is, $\partial_{\omega}\left(A_{\aleph}, B_{\aleph}\right)=\partial_{\omega}\left(B_{\aleph}, A_{\aleph}\right)$

(b) the correlation $\partial_{\omega}$ finds the interval $[-1,1]$, that is, $-1 \leq \partial_{\omega}\left(A_{\aleph}, B_{\aleph}\right) \leq 1$

(c) $\partial_{\omega}\left(A_{\aleph}, B_{\aleph}\right)=1$ if $\left.A_{\aleph}=(\lambda)\right) B_{\aleph}, \lambda>0$

(here, $(\lambda) B_{\aleph}$ means $\left\{\left\langle\left[\lambda \zeta_{i j}^{-}, \lambda \zeta_{i j}^{+}\right], \lambda \zeta_{i j}\right\rangle_{j=1}^{m}\right)$

(d) $\partial_{\omega}\left(A_{\aleph}, B_{\aleph}\right)=1$ if $A_{\aleph}=B_{\aleph}$

\section{Proof}

(a) Obvious.

(b) By Proposition 4, we have

$$
\begin{aligned}
& \left|{ }^{\omega} I_{j\left(A_{\aleph} \longrightarrow B_{\aleph}\right)}^{-}\right| \leq \sqrt{{ }^{\omega} \mathbb{C}_{j}^{-}\left(A_{\aleph}\right)^{\omega} \mathbb{C}_{j}^{-}\left(B_{\aleph}\right)} \\
& \Rightarrow-\sqrt{{ }^{\omega} \mathbb{C}_{j}^{-}\left(A_{\aleph}\right){ }^{\omega} \mathbb{C}_{j}^{-}\left(B_{\aleph}\right)} \leq{ }^{\omega} I_{j}^{-}\left(A_{\aleph} \longrightarrow B_{\aleph}\right) \leq \sqrt{{ }^{\omega} \mathbb{C}_{j}^{-}\left(A_{\aleph}\right){ }^{\omega} \mathbb{C}_{j}^{-}\left(B_{\aleph}\right)} \\
& \left.\Rightarrow-1 \leq{ }^{\omega} I_{j\left(A_{\aleph}\right.}^{-} \rightarrow B_{\aleph}\right) \sqrt{{ }^{\omega} \mathbb{C}_{j}^{-}\left(A_{\aleph}\right){ }^{\omega} \mathbb{C}_{j}^{-}\left(B_{\aleph}\right)} \leq 1 \\
& \Rightarrow-1 \leq \frac{\sum_{i=1}^{n} \omega_{i}\left(\eta_{i j}^{-}-\overline{\eta_{j}}\right)\left(\zeta_{i j}^{-}-\overline{\zeta_{j}^{-}}\right)}{\sqrt{\left.\sum_{i=1}^{n} \omega_{i}\left(\eta_{i j}^{-}-\overline{\left(\eta_{j}^{-}\right.}\right)\right)^{2} \sum_{i=1}^{n} \omega_{i}\left(\zeta_{i j}^{-}-\overline{\left(\zeta_{j}^{-}\right)}\right)^{2}}} \leq 1 \\
& \Rightarrow-1 \leq{ }^{\omega} \partial_{j}^{-}\left(A_{\aleph}, B_{\aleph} \leq 1\right. \text {. }
\end{aligned}
$$

(c) Suppose $A_{\aleph}=(\lambda) B_{\aleph}$. Then, $\eta_{i j}^{-}=\lambda \zeta_{i j}^{-}, \eta_{i j}^{+}=\lambda \zeta_{i j}^{+}$, and $\eta_{i j}=\lambda \zeta_{i j}$. In this case,

$$
\begin{aligned}
{ }^{\omega} \partial_{j}^{-}\left(A_{\aleph}, B_{\aleph}\right) & =\frac{\sum_{i=1}^{n} \omega_{i}\left(\overline{\eta_{i j}^{-}}-\overline{\eta_{j}^{-}}\right)\left(\zeta_{i j}^{-}-\overline{\zeta_{j}}\right)}{\sqrt{\sum_{i=1}^{n} \omega_{i}\left(\overline{\eta_{i j}^{-}}-\overline{\left(\eta_{j}^{-}\right)}\right)^{2} \sum_{i=1}^{n} \omega_{i}\left(\zeta_{i j}^{-}-\overline{\left(\zeta_{j}^{-}\right)}\right)^{2}}} \\
& =\frac{\sum_{i=1}^{n} \omega_{i}\left(\lambda \zeta_{i j}^{-}-\lambda \overline{\zeta_{j}^{-}}\right)\left(\zeta_{i j}^{-}-\overline{\zeta_{j}^{-}}\right)}{\sqrt{\sum_{i=1}^{n} \omega_{i}\left(\lambda \zeta_{i j}^{-}-\lambda \overline{\left(\zeta_{j}^{-}\right)}\right)^{2} \sum_{i=1}^{n} \omega_{i}\left(\zeta_{i j}^{-}-\overline{\left(\zeta_{j}^{-}\right)}\right)^{2}}} \\
& =\frac{\sum_{i=1}^{n} \omega_{i}\left(\zeta_{i j}^{-}-\overline{\zeta_{j}^{-}}\right)^{2}}{\sqrt{\sum_{i=1}^{n} \omega_{i}\left(\zeta_{i j}^{-}-\overline{\left(\zeta_{j}^{-}\right)}\right)^{2} \sum_{i=1}^{n} \omega_{i}\left(\zeta_{i j}^{-}-\overline{\left(\zeta_{j}^{-}\right)}\right)^{2}}}=1 .
\end{aligned}
$$

Similarly, ${ }^{\omega} \partial_{j}^{+}\left(A_{\aleph}, B_{\aleph}\right)=1,{ }^{\omega} \partial_{j}\left(A_{\aleph}, B_{\aleph}\right)=1$, and hence, $\partial_{\omega}\left(A_{\aleph}, B_{\aleph}\right)=1$, being the average of $1^{\prime} s$.

(d) is similar to (c).

Definition 18. For the CmPFSs $A_{\aleph}$ and $B_{\aleph}$, the WCC may also be defined as

$$
\sigma_{\omega}\left(A_{\aleph}, B_{\aleph}\right)=\frac{1}{3 m}\left(\sum_{j=1}^{m}{ }^{\omega} \sigma_{j}^{-}\left(A_{\aleph}, B_{\aleph}\right)+\sum_{j=1}^{m}{ }^{\omega} \sigma_{j}^{+}\left(A_{\aleph}, B_{\aleph}\right)+\sum_{j=1}^{m}{ }^{\omega} \sigma_{j}\left(A_{\aleph}, B_{\aleph}\right)\right),
$$


where

$$
\begin{aligned}
{ }^{\omega} \sigma_{j}^{-}\left(A_{\aleph}, B_{\aleph}\right) & =\frac{\sum_{i=1}^{n} \omega_{i}\left(\eta_{i j}^{-}-\overline{\eta_{j}^{-}}\right)\left(\zeta_{i j}^{-}-\overline{\zeta_{j}^{-}}\right)}{\max \left\{\sum_{i=1}^{n} \omega_{i}\left(\eta_{i j}^{-}-\overline{\left(\eta_{j}^{-}\right)}\right)^{2}, \sum_{i=1}^{n} \omega_{i}\left(\zeta_{i j}^{-}-\overline{\left(\zeta_{j}^{-}\right)}\right)^{2}\right\}}, \\
{ }^{\omega} \sigma_{j}^{+}\left(A_{\aleph}, B_{\aleph}\right) & =\frac{\sum_{i=1}^{n} \omega_{i}\left(\eta_{i j}^{+}-\overline{\eta_{j}^{+}}\right)\left(\zeta_{i j}^{+}-\overline{\zeta_{j}^{+}}\right)}{\max \left\{\sum_{i=1}^{n} \omega_{i}\left(\eta_{i j}^{+}-\overline{\left(\eta_{j}^{+}\right)}\right)^{2}, \sum_{i=1}^{n} \omega_{i}\left(\zeta_{i j}^{+}-\overline{\left(\zeta_{j}^{+}\right)}\right)^{2}\right\}}, \\
{ }^{\omega} \sigma_{j}\left(A_{\aleph}, B_{\aleph}\right) & =\frac{\sum_{i=1}^{n} \omega_{i}\left(\eta_{i j}-\overline{\eta_{j}}\right)\left(\zeta_{i j}-\overline{\zeta_{j}}\right)}{\max \left\{\sum_{i=1}^{n} \omega_{i}\left(\eta_{i j}-\overline{\left(\eta_{j}\right)}\right)^{2}, \sum_{i=1}^{n} \omega_{i}\left(\zeta_{i j}-\overline{\left(\zeta_{j}\right)}\right)^{2}\right\}},
\end{aligned}
$$

$j=1,2, \ldots, m$.

Theorem 15. If $A_{\aleph}$ and $B_{\aleph}$ are CmPFSs which are dictated by the weights, then

(a) the correlation $\sigma_{\omega}$ is symmetrical, that is, $\sigma_{\omega}\left(A_{\aleph}, B_{\aleph}\right)=\sigma_{\omega}\left(B_{\aleph}, A_{\aleph}\right)$

(b) the correlation $\sigma_{\omega}$ finds the interval $[-1,1]$, that is, $-1 \leq \sigma_{\omega}\left(A_{\aleph}, B_{\aleph}\right) \leq 1$

(c) $\sigma_{\omega}\left(A_{\aleph}, B_{\aleph}\right)=1$ if $A_{\aleph}=B_{\aleph}$
Proof. The proof is obvious.

The two types of the weighted correlation coefficients are equally effective no matter what types of weights are assigned to the alternatives. However, the weights should follow the mentioned conditions. If there are " $n$ " alternatives and are affected by the weights $((1 / n),(1 / n), \ldots,(1 / n)$, then we see that

$$
\begin{aligned}
& { }^{\omega} \partial_{j}^{-}\left(A_{\aleph}, B_{\aleph}\right)=\frac{\sum_{i=1}^{n} \omega_{i}\left(\eta_{i j}^{-}-\overline{\eta_{j}^{-}}\right)\left(\zeta_{i j}^{-}-\overline{\zeta_{j}^{-}}\right)}{\sqrt{\sum_{i=1}^{n} \omega_{i}\left(\eta_{i j}^{-}-\overline{\left(\eta_{j}^{-}\right)}\right)^{2} \sum_{i=1}^{n} \omega_{i}\left(\zeta_{i j}^{-}-\overline{\left.\left(\zeta_{j}^{-}\right)\right)^{2}}\right.}} \\
& =\frac{\sum_{i=1}^{n}(1 / n)\left(\eta_{i j}^{-}-\overline{\eta_{j}^{-}}\right)\left(\zeta_{i j}^{-}-\overline{\zeta_{j}^{-}}\right)}{\sqrt{\sum_{i=1}^{n}(1 / n)\left(\eta_{i j}^{-}-\overline{\left(\eta_{j}^{-}\right)}\right)^{2} \sum_{i=1}^{n}(1 / n)\left(\zeta_{i j}^{-}-\overline{\left(\zeta_{j}^{-}\right)}\right)^{2}}} \\
& =\frac{\sum_{i=1}^{n}\left(\eta_{i j}^{-}-\overline{\eta_{j}^{-}}\right)\left(\zeta_{i j}^{-}-\overline{\zeta_{j}^{-}}\right)}{\sqrt{\sum_{i=1}^{n}\left(\eta_{i j}^{-}-\overline{\left(\eta_{j}^{-}\right)}\right)^{2} \sum_{i=1}^{n}\left(\zeta_{i j}^{-}-\overline{\left(\zeta_{j}^{-}\right)}\right)^{2}}}=\partial_{j}^{-}\left(A_{\aleph}, B_{\aleph}\right), \\
& { }^{\omega} \sigma_{j}^{-}\left(A_{\aleph}, B_{\aleph}\right)=\frac{\sum_{i=1}^{n} \omega_{i}\left(\eta_{i j}^{-}-\overline{\eta_{j}^{-}}\right)\left(\zeta_{i j}^{-}-\overline{\zeta_{j}^{-}}\right)}{\left.\max \left\{\sum_{i=1}^{n} \omega_{i}\left(\eta_{i j}^{-}-\overline{\left(\eta_{j}^{-}\right)}\right)^{2}, \sum_{i=1}^{n} \omega_{i}\left(\zeta_{i j}^{-}-\overline{\left(\zeta_{j}^{-}\right.}\right)\right)^{2}\right\}} \\
& =\frac{\sum_{i=1}^{n}(1 / n)\left(\eta_{i j}^{-}-\overline{\eta_{j}^{-}}\right)\left(\zeta_{i j}^{-}-\overline{\zeta_{j}^{-}}\right)}{\max \left\{\sum_{i=1}^{n}(1 / n)\left(\eta_{i j}^{-}-\overline{\left(\eta_{j}^{-}\right)}\right)^{2}, \sum_{i=1}^{n}(1 / n)\left(\zeta_{i j}^{-}-\overline{\left(\zeta_{j}^{-}\right)}\right)^{2}\right\}} \\
& =\frac{\sum_{i=1}^{n}\left(\eta_{i j}^{-}-\overline{\eta_{j}^{-}}\right)\left(\zeta_{i j}^{-}-\overline{\zeta_{j}^{-}}\right)}{\max \left\{\sum_{i=1}^{n}\left(\eta_{i j}^{-}-\overline{\left(\eta_{j}^{-}\right)}\right)^{2}, \sum_{i=1}^{n}\left(\zeta_{i j}^{-}-\overline{\left(\zeta_{j}^{-}\right)}\right)^{2}\right\}}=\sigma_{j}^{-}\left(A_{\aleph}, B_{\aleph}\right),
\end{aligned}
$$

and similarly others. This shows that the above defined correlations are invariant under the weights $((1 / n)$, $(1 / n), \ldots,(1 / n)$. So, whenever we are encountered by such weights, we can simply ignore them and proceed in an unweighted manner. That is, the weighted alternatives can be treated as unweighted ones. 
Step 1: construct the pattern $\widetilde{\mathfrak{P}}$ which is to be categorized.

Step 2: assemble the known patterns $\widetilde{\mathfrak{P}}^{(1)}, \widetilde{\mathfrak{P}}^{(2)}, \ldots, \widetilde{\mathfrak{P}}^{(\mathfrak{t})}$, over a discourse $\aleph$, among which the recognition is to be performed. Step 3 (a): find the correlation coefficients of the pattern $\widetilde{\mathfrak{P}}$ from the known patterns $\widetilde{\mathfrak{P}}^{(1)}, \widetilde{\mathfrak{P}}^{(2)}, \ldots, \widetilde{\mathfrak{P}}^{(\mathfrak{P})}$ using formula (34) or (39). Step 3 (b): if the alternatives from the discourse $\aleph$ follow some weights, then find the respective weighted correlation coefficients using formula (46) or (50).

Step 4: if the pattern $\widetilde{\mathfrak{P}}^{(\mathrm{i})}$ finds the greatest correlation with $\widetilde{\mathfrak{P}}$, then the pattern $\widetilde{\mathfrak{P}}$ belongs to $\widetilde{\mathfrak{P}}^{(\mathrm{i})}$.

Algorithm 1: Steps of the proposed approach.

\section{Applications of the Proposed Correlation Measures}

Correlation actions are common indicators and effective approaches to address MCDM problems. In different uncertain environments, various scholars have used correlations to solve such problems efficiently and effectively. The correlations defined in this article are used here in this section to accurately recognize the patterns and medical diagnosis. Pattern recognition (PR) finds its roots in machine learning and statistics. Due to the availability of big data, the recognition problems are subjected to automatization. Bishop [38] gave a modern definition of PR as, "The field of pattern recognition is handled with the automated design of swings in data through the use of computer algorithms and with the use of these rhythms to take operations such as ordering the data into separate classes [38].” PR algorithms commonly endeavor to present a sensible answer for all attainable inputs and to achieve "most likely" matching of the inputs, using the statement of their statistical variation. They are classified according to the variety of learning procedures used to produce the output value.

5.1. Illustrative Examples. In the following example, we use our proposed correlations to categorize an unknown pattern in $\mathrm{CmPF}$ environment (Algorithm 1).
The flowchart for the proposed algorithm is demonstrated in Figure 2.

Example 7. In this example, we give a solution to a pattern recognition problem. Suppose a mining firm discovers a new type of carbon while working on a site. The experts analyze it completely keeping in view its structure, properties, and smell and give their opinion about its pattern. According to the expert's point of view, suppose its pattern comes out to be a cubic 3-polar fuzzy set over the discourse $\aleph=\left\{\kappa_{1}, \kappa_{2}, \kappa_{3}\right\}$, given by

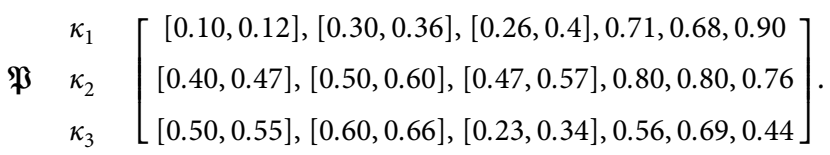

The experts are aware that there are three patterns (allotropes) of carbon, that is, diamond, graphite, and fullerenes, which are already known. Now, the problem is to identify the discovered pattern among the three already known ones. For this purpose, the three known allotropes are reanalyzed and the same type of CmPF patterns is assigned to them:

$$
\begin{aligned}
& \begin{array}{cc} 
& \kappa_{1} \\
\left.A_{\aleph}(\text { diamond })\right) \widetilde{\mathfrak{P}}^{(1)} & \kappa_{2} \\
& \kappa_{3}
\end{array} \quad\left[\begin{array}{l}
{[0.20,0.40],[0.29,0.48],[0.32,0.55], 0.57,0.66,0.42} \\
{[0.32,0.58],[0.25,0.39],[0.48,0.56], 0.60,0.50,0.40} \\
{[0.49,0.62],[0.31,0.43],[0.36,0.48], 0.20,0.35,0.50}
\end{array}\right],
\end{aligned}
$$

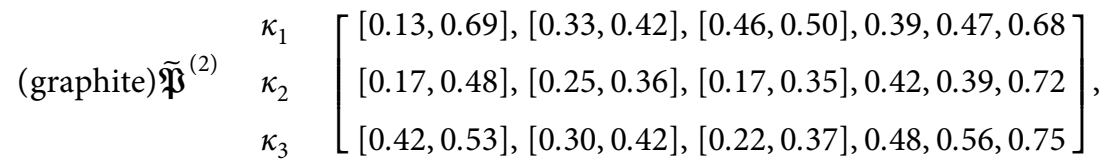

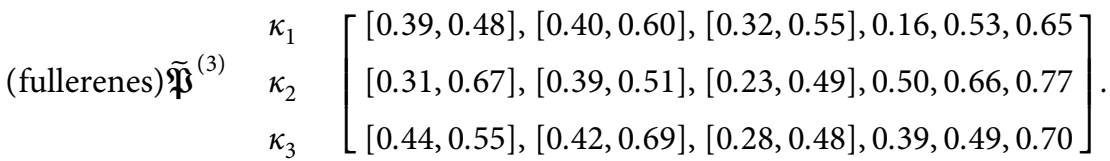




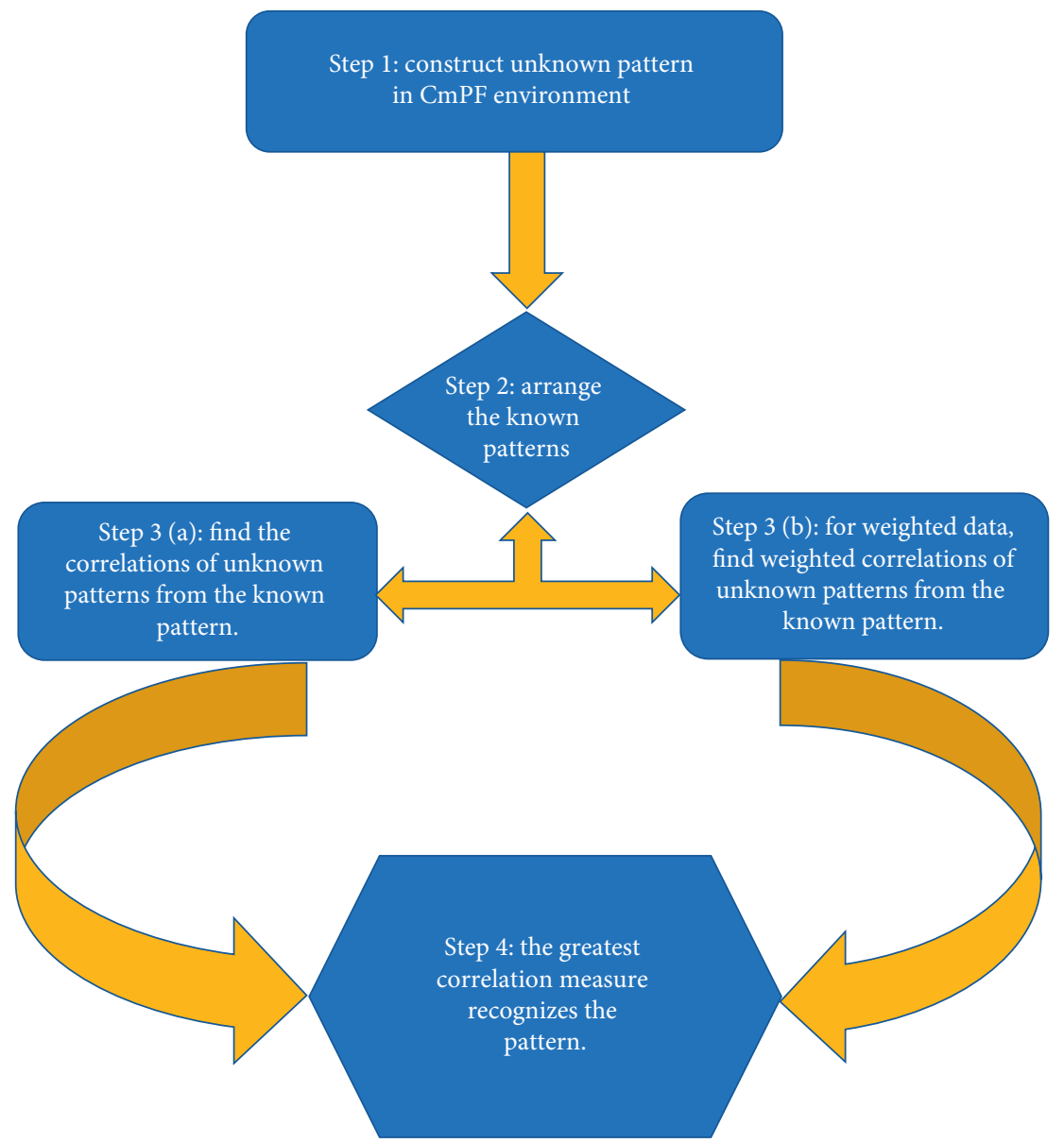

FIGURE 2: Flowchart for the proposed algorithm.

We find the correlations of $\mathfrak{P}$ from $\widetilde{\mathfrak{P}}^{(1)}, \widetilde{\mathfrak{P}}^{(2)}, \widetilde{\mathfrak{P}}^{(3)}$ (using formulae (34) or (39), respectively) as follows:

$$
\begin{aligned}
& \partial\left(\mathfrak{P}, \widetilde{\mathfrak{P}}^{(1)}\right)=0.33, \\
& \partial\left(\mathfrak{P}, \widetilde{\mathfrak{P}}^{(2)}\right)=-0.49, \\
& \partial\left(\mathfrak{P}, \widetilde{\mathfrak{P}}^{(3)}\right)=0.16, \\
& \sigma\left(\mathfrak{P}, \widetilde{\mathfrak{P}}^{(1)}\right)=0.24, \\
& \sigma\left(\mathfrak{P}, \widetilde{\mathfrak{P}}^{(2)}\right)=-0.20, \\
& \sigma\left(\mathfrak{P}, \widetilde{\mathfrak{P}}^{(3)}\right)=0.10 .
\end{aligned}
$$

This shows that $\mathfrak{P}$ finds the greatest correlations with $\widetilde{\mathfrak{P}}^{(1)}$, so the pattern $\mathfrak{P}$ belongs to $\widetilde{\mathfrak{P}}^{(1)}$.

However, if the alternatives $\kappa_{1}, \kappa_{2}, \kappa_{3}$ follow the weights $\omega=(0.29,0.33,0.38)$, then we find the respective weighted correlations (using formulae (46) and (50), respectively) as follows:

$$
\begin{aligned}
& { }^{\omega} \partial\left(\mathfrak{P}, \widetilde{\mathfrak{P}}^{(1)}\right)=0.35, \\
& { }^{\omega} \partial\left(\mathfrak{P}, \widetilde{\mathfrak{P}}^{(2)}\right)=-0.47, \\
& { }^{\omega} \partial\left(\mathfrak{P}, \widetilde{\mathfrak{P}}^{(3)}\right)=0.18, \\
& { }^{\omega} \partial\left(\mathfrak{P}, \widetilde{\mathfrak{P}}^{(1)}\right)=0.24, \\
& { }^{\omega} \partial\left(\mathfrak{P}, \widetilde{\mathfrak{P}}^{(2)}\right)=-0.19, \\
& { }^{\omega} \partial\left(\mathfrak{P}, \widetilde{\mathfrak{P}}^{(3)}\right)=0.11 .
\end{aligned}
$$

The same result is obtained using the weighted correlations. Hence, our proposed correlations are consistent with each other.

Example 8. Suppose a patient $\mathrm{P}$ is to be diagnosed in a medical center. A medical team examines him completely, and the following symptoms are observed in the patient:

$$
\mathrm{S}=\left\{\left(\kappa_{1} \text {, headache }\right),\left(\kappa_{2}, \text { cough }\right),\left(\kappa_{3} \text {, chest pain }\right),\left(\kappa_{4} \text {, shortness of breath }\right)\right\} .
$$


TABLE 2: Rating values of the patient in the CmPFS environment.

\begin{tabular}{lr}
\hline $\mathrm{P}$ & Patient \\
\hline$\kappa_{1}$ & {$[0.20,0.40],[0.18,0.39],[0.15,0.27], 0.69,0.72,0.88$} \\
$\kappa_{2}$ & {$[0.42,0.60],[0.30,0.50],[0.19,0.33], 0.40,0.60,0.80$} \\
$\kappa_{3}$ & {$[0.55,0.62],[0.42,0.55],[0.18,0.25], 0.12,0.27,0.39$} \\
$\kappa_{4}$ & {$[0.49,0.69],[0.52,0.64],[0.42,0.49], 0.43,0.55,0.67$} \\
\hline
\end{tabular}

TABLE 3: Rating values of each diagnosis in the CmPFS environment.

\begin{tabular}{lc}
\hline$\Upsilon_{1}$ & Corona \\
\hline$\kappa_{1}$ & {$[0.32,0.44],[0.22,0.27],[0.09,0.27], 0.42,0.53,0.64$} \\
$\kappa_{2}$ & {$[0.14,0.26],[0.30,0.39],[0.42,0.51], 0.48,0.59,0.68$} \\
$\kappa_{3}$ & {$[0.16,0.43],[0.33,0.40],[0.32,0.47], 0.20,0.30,0.40$} \\
$\kappa_{4}$ & {$[0.21,0.33],[0.49,0.56],[0.40,0.50], 0.17,0.39,0.44$} \\
\hline$\Upsilon_{2}$ & Diarrhea \\
\hline$\kappa_{1}$ & {$[0.16,0.24],[0.23,0.36],[0.27,0.39], 0.39,0.47,0.55$} \\
$\kappa_{2}$ & {$[0.04,0.17],[0.14,0.24],[0.37,0.47], 0.40,0.45,0.60$} \\
$\kappa_{3}$ & {$[0.24,0.36],[0.39,0.47],[0.45,0.57], 0.37,0.49,0.54$} \\
$\kappa_{4}$ & {$[0.32,0.48],[0.42,0.54],[0.40,0.51], 0.25,0.27,0.33$} \\
\hline$\Upsilon_{3}$ & Asthma \\
\hline$\kappa_{1}$ & {$[0.31,0.43],[0.40,0.55],[0.60,0.71], 0.41,0.39,0.55$} \\
$\kappa_{2}$ & {$[0.24,0.36],[0.26,0.40],[0.42,0.49], 0.47,0.56,0.63$} \\
$\kappa_{3}$ & {$[0.13,0.28],[0.09,0.13],[0.37,0.47], 0.44,0.53,0.72$} \\
$\kappa_{4}$ & {$[0.15,0.33],[0.29,0.37],[0.36,0.56], 0.80,0.90,0.70$} \\
\hline$\Upsilon_{4}$ & Sore throat \\
\hline$\kappa_{1}$ & {$[0.41,0.54],[0.29,0.47],[0.68,0.73], 0.39,0.48,0.57$} \\
$\kappa_{2}$ & {$[0.38,0.59],[0.39,0.47],[0.40,0.51], 0.41,0.47,0.56$} \\
$\kappa_{3}$ & {$[0.62,0.80],[0.55,0.65],[0.32,0.46], 0.62,0.55,0.39$} \\
$\kappa_{4}$ & {$[0.40,0.70],[0.38,0.42],[0.62,0.70], 0.60,0.72,0.54$} \\
\hline
\end{tabular}

The set of diagnoses which the patient can observe is given by $\Upsilon_{1}$ (corona), $\Upsilon_{2}$ (diarrhea), $\Upsilon_{3}$ (asthma), $\Upsilon_{4}$ (sore throat). Suppose the expert's point of views about the patient, taking these symptoms into account, comes out to be a CmPFS (Table 2).

Therefore, each diagnosis must also be viewed as CmPFS, given by Table 3 .

Our aim is to find that to which disease the patient belongs, that is, to examine that from which disease the patient is suffering from. For this purpose, we find the following correlation coefficients (using formulae (34) or (39), respectively):

$$
\begin{aligned}
& \partial\left(P, \Upsilon_{1}\right)=0.42 \\
& \partial\left(P, \Upsilon_{2}\right)=0.33 \\
& \partial\left(P, \Upsilon_{3}\right)=-0.50 \\
& \partial\left(P, \Upsilon_{4}\right)=0.28 \\
& \sigma\left(P, \Upsilon_{1}\right)=0.35 \\
& \sigma\left(P, \Upsilon_{2}\right)=0.27 \\
& \sigma\left(P, \Upsilon_{3}\right)=-0.31 \\
& \sigma\left(P, \Upsilon_{4}\right)=0.21
\end{aligned}
$$

However, if the symptoms bear some credibility weights $\omega=(0.17,0.18,0.25,0.40)$, then we find the respective weighted correlations (using formulae (46) and (50), respectively) as follows:

$$
\begin{gathered}
{ }^{\omega} \partial\left(\mathrm{P}, \Upsilon_{1}\right)=0.42, \\
{ }^{\omega} \partial\left(\mathrm{P}, \Upsilon_{2}\right)=0.35, \\
{ }^{\omega} \partial\left(\mathrm{P}, \Upsilon_{3}\right)=-0.43, \\
{ }^{\omega} \partial\left(\mathrm{P}, \Upsilon_{4}\right)=0.29, \\
{ }^{\omega} \partial\left(\mathrm{P}, \Upsilon_{1}\right)=0.37, \\
{ }^{\omega} \partial\left(\mathrm{P}, \Upsilon_{2}\right)=0.28, \\
{ }^{\omega} \partial\left(\mathrm{P}, \Upsilon_{3}\right)=-0.24, \\
{ }^{\omega} \partial\left(\mathrm{P}, \Upsilon_{4}\right)=0.22 .
\end{gathered}
$$

We have used all of the four proposed correlations, and we found $\Upsilon_{1}$ as optimal solution. Hence, the patient $P$ is suffering from corona.

\section{Conclusion}

The paper aims to present the novel correlation coefficient between the pairs of the cubic $m$-polar fuzzy sets. The considered CmPFS is a more integrated set to handle the uncertainties in the data than the existing $m$-polar fuzzy set and the cubic set. The advantage of the CmPFS over these existing sets is that it represents the information about the element by using $m$ fuzzy intervals as well as $m$ fuzzy numbers. Keeping these advantages, we investigate some operational laws about the CmPFS and hence investigate their properties. Furthermore, we elaborate on the information energy and hence define the correlation coefficient between the pairs of CmPFS. The major advantage of the proposed measure is that it considered the range of correlation measures as $[-1,1]$ instead of $[0,1]$ only. Therefore, the proposed measure has a stronger measure of the negative relationship between the variables also. Furthermore, the applicability of the proposed measures has been demonstrated through a case study of pattern recognition as well as medical diagnosis problems. The results of the proposed method are compared with several existing approaches which reveal the effectiveness of the presented work. In future studies, we shall study applications of the proposed methodology under the different fuzzy environment and solve some more practical problems [39-45].

\section{Data Availability}

Data sharing is not applicable to this article as no datasets were generated or analysed during the current study.

\section{Conflicts of Interest}

The authors declare that they have no conflicts of interest. 


\section{References}

[1] L. A. Zadeh, "Fuzzy sets," Information and Control, vol. 8, no. 3, pp. 338-353, 1965.

[2] W. R. Wen-Ran Zhang, "Bipolar fuzzy sets and relations: a computational framework for cognitive modeling and multiagent decision analysis," in NAFIPS/IFIS/NASA '94. Proceedings of the First International Joint Conference of The North American Fuzzy Information Processing Society Biannual Conference. The Industrial Fuzzy Control and Intelligent Systems Conference, and the NASA Joint Technology Wo, San Antoni, TX, USA, December 1995.

[3] K. T. Atanassov, "Intuitionistic fuzzy sets," Fuzzy Sets and Systems, vol. 20, no. 1, pp. 87-96, 1986.

[4] J. Chen, S. Li, S. Ma, and X. Wang, "m-Polar Fuzzy sets: an extension of bipolar fuzzy sets," The Scientific World Journal, vol. 2014, Article ID 416530, 2014.

[5] Y. B. Jun, C. S. Kim, and K. O. Yang, "Cubic sets," Annals of Fuzzy Mathematics and Informatics, vol. 4, no. 1, pp. 83-98, 2012.

[6] M. Riaz and M. R. Hashmi, "MAGDM for agribusiness in the environment of various cubic m-polar fuzzy averaging aggregation operators," Journal of Intelligent \& Fuzzy Systems, vol. 37, no. 3, pp. 3671-3691, 2019.

[7] R. R. Yager, "Pythagorean fuzzy subsets," in Proceedings of the 2013 Joint IFSA World Congress and NAFIPS Annual Meeting (IFSA/NAFIPS), pp. 57-61, IEEE, Edmonton, Canada, June 2013.

[8] R. R. Yager, "Generalized orthopair fuzzy sets," IEEE Transactions on Fuzzy Systems, vol. 25, no. 5, pp. 1220-1230, 2017.

[9] F. Smarandache, "A unifying field in logics: neutrosophic logic," Neutrosophy, Neutrosophic Set, Neutrosophic Probability and Statistics, American Research Press, Santa Fe, NM, USA, 1999.

[10] H. Wang, F. Smarandache, Y. Q. Zhang, and R. Sunderraman, "Single valued neutrosophic sets," Multispace and Multistructure, vol. 4, pp. 410-413, 2010.

[11] S. Ashraf and S. Abdullah, "Spherical aggregation operators and their application in multiattribute group decision-making," International Journal of Intelligent Systems, vol. 34, no. 3, pp. 493-523, 2019.

[12] F. K. Gündoğdu and C. Kahraman, "Spherical fuzzy sets and spherical fuzzy TOPSIS method," Journal of Intelligent \& Fuzzy Systems, vol. 36, no. 1, pp. 337-352, 2019.

[13] T. Mahmood, K. Ullah, Q. Khan, and N. Jan, "An approach toward decision-making and medical diagnosis problems using the concept of spherical fuzzy sets," Neural Computing and Applications, vol. 31, no. 11, pp. 7041-7053, 2019.

[14] B. C. Cuong, "Picture fuzzy sets-first results," in Preprint of Seminar on Neuro-Fuzzy Systems with ApplicationsInstitute of Mathematics, Hanoi, Vietnam, 2013.

[15] D. Molodtsov, "Soft set theory-first results," Computers and Mathematics with Applications, vol. 37, Article ID 199631, 1999.

[16] L. Wang, H. Garg, and N. Li, "Pythagorean fuzzy interactive Hamacher power aggregation operators for assessment of express service quality with entropy weight," Soft Computing, vol. 25, pp. 973-993, 2021.

[17] Y. Liu, G. Wei, S. Abdullah, J. Liu, L. Xu, and H. Liu, "Banzhaf-Choquet-copula-based aggregation operators for managing q-rung orthopair fuzzy information," Soft Computing, vol. 25, no. 10, pp. 6891-6914, 2021.
[18] M. Akram, A. Khan, J. C. R. Alcantud, and G. Santos-Garcia, "A hybrid decision-making framework under complex spherical fuzzy prioritized weighted aggregation operators," Expert Systems, vol. 46, no. 3, 2021.

[19] M. Sitara, M. Akram, and M. Riaz, "Decision-making analysis based on q-rung picture fuzzy graph structures," Journal of Applied Mathematics and Computing, vol. 14, no. 2, 2021.

[20] H. Garg, "Multi-attribute group decision-making process based on possibility degree and operators for intuitionistic multiplicative set," Complex \& Intelligent Systems, vol. 7, no. 2, pp. 1099-1121, 2021.

[21] H. Garg, Z. Ali, and T. Mahmood, "Interval-valued picture uncertain linguistic generalized hamacher aggregation operators and their application in multiple attribute decisionmaking process," Arabian Journal For Science And Engineering, vol. 31, 2021.

[22] P. Liu, Z. Ali, T. Mahmood, and N. Hassan, "Group decisionmaking using complex q-rung orthopair fuzzy Bonferroni mean," International Journal of Computational Intelligence Systems, vol. 13, no. 1, pp. 822-851, 2020.

[23] P. Liu and P. Wang, "Multiple attribute group decision making method based on intuitionistic fuzzy Einstein interactive operations," International Journal of Fuzzy Systems, vol. 22, no. 3, pp. 790-809, 2020.

[24] M. Riaz and M. R. Hashmi, "Linear Diophantine fuzzy set and its applications towards multi-attribute decision-making problems," Journal of Intelligent \& Fuzzy Systems, vol. 37, no. 4, pp. 5417-5439, 2019.

[25] M. Riaz, M. Raza Hashmi, D. Pamucar, and Y. Chu, "Spherical linear Diophantine fuzzy sets with modeling uncertainties in MCDM," Computer Modeling in Engineering \& Sciences, vol. 126, no. 3, pp. 1125-1164, 2021.

[26] H. Kamac1, "Linear Diophantine fuzzy algebraic structures," Journal of Ambient Intelligence and Humanized Computing, vol. 22, no. 3, 2021.

[27] S. Ayub, M. Shabir, M. Riaz, M. Aslam, and R. Chinram, "Linear diophantine fuzzy relations and their algebraic properties with decision making," Symmetry, vol. 13, no. 6, pp. 1-18, 2021.

[28] A. O. Almagrabi, S. Abdullah, and M. Shams, "A new approach to q-linear Diophantine fuzzy emergency decision support system for COVID-19," Journal of Ambient Intelligence and Humanoized Computing, vol. 50, no. 1, 2021.

[29] A. H. Ganie and S. Singh, "A picture fuzzy similarity measure based on direct operations and novel multi-attribute decisionmaking method," Neural Computing and Applications, vol. 33, no. 15, pp. 9199-9219, 2021.

[30] T. Mahmood and Z. Ali, "Entropy measure and TOPSIS method based on correlation coefficient using complex q-rung orthopair fuzzy information and its application to multiple attribute decision making," Soft Computing, vol. 25, no. 3, pp. 1249-1275, 2021.

[31] R. M. Zulqarnain, I. Siddique, F. Jarad, R. Ali, and T. Abdeljawad, "Development of TOPSIS technique under Pythagorean fuzzy hypersoft environment based on correlation coefficient and its application towards the selection of antivirus mask in COVID-19 pandemic," Complexity, vol. 2021, pp. 1-27, 2021.

[32] P. A. Ejegwa, I. C. Onyeke, and V. Adah, "An algorithm for an improved intuitionistic fuzzy correlation measure with medical diagnostic application," Annals of Optimization Theory and Practice, vol. 3, no. 3, pp. 51-66, 2020. 
[33] T. Gerstenkorn and J. Mańko, "Correlation of intuitionistic fuzzy sets," Fuzzy Sets and Systems, vol. 44, no. 1, pp. 39-43, 1991.

[34] E. Szmidt and J. Kacprzyk, "Correlation of intuitionistic fuzzy sets, computational intelligence for knowledge-based systems design. IPMU," Lecture Notes in Computer Science, Springer, Berlin, Heidelberg, 2010.

[35] H. Garg and G. Kaur, "Algorithm for solving the decisionmaking problems based on correlation coefficients under cubic intuitionistic fuzzy information: a case study in watershed hydrological system," Complex \& Intelligent Systems, vol. 33, pp. 1-20, 2021.

[36] M. Lin, C. Huang, R. Chen, H. Fujita, and X. Wang, "Directional correlation coefficient measures for Pythagorean fuzzy sets: their applications to medical diagnosis and cluster analysis," Complex \& Intelligent Systems, vol. 7, no. 2, pp. 1025-1043, 2021.

[37] N. X. Thao, "A new correlation coefficient of the Pythagorean fuzzy sets and its applications," Soft Computing, vol. 24, no. 13, pp. 9467-9478, 2020.

[38] C. M. Bishop, Pattern Recognition and Machine Learning, Springer, Berlin, Germany, 2006.

[39] K. Ullah, T. Mahmood, N. Jan, and Z. Ahmad, "Policy decision making based on some averaging aggregation operators of T-spherical fuzzy sets; a multi-attribute decision making approach," Annals of Optimization Theory \& Practices, vol. 3, no. 3, pp. 69-92, 2020.

[40] C. Koukoumis and A. Karagrigoriou, "On entropy-type measures and divergences with applications in engineering, management and applied sciences," International Journal of Mathematical, Engineering and Management Sciences, vol. 6, no. 3, pp. 688-707, 2021.

[41] P. Talukdar, S. Goala, P. Dutta, and B. Limboo, "Fuzzy multicriteria decision making in medical diagnosis using an advanced distance measure on linguistic Pythagorean fuzzy sets," Annals of Optimization Theory \& Practices, vol. 3, no. 4, pp. 113-131, 2020.

[42] G. Goyal and D. C. S. Bisht, "Sugeno intuitionistic fuzzy generator based computational technique for crude oil price forecasting," International Journal of Mathematical, Engineering and Management Sciences, vol. 5, no. 3, pp. 488-496, 2020.

[43] A. Fahmi, F. Amin, and S. B. H. Shah, "Geometric operators based on linguistic interval-valued intuitionistic neutrosophic fuzzy number and their application in decision making," Annals of Optimization Theory \& Practices, vol. 3, no. 1, pp. 47-61, 2020.

[44] M. E. Alaoui, "A fuzzy multiplicative performance indicator to measure circular economy efficiency," International Journal of Mathematical, Engineering and Management Sciences, vol. 5, no. 6, pp. 1118-1127, 2020.

[45] H. Garg, "New exponential operation laws and operators for interval-valued q-rung orthopair fuzzy sets in group decision making process," Neural Computing and Applications, vol. 36, pp. 1-27, 2021. 